\title{
A multiscale approach to modelling electrochemical processes occurring across the cell membrane with application to transmission of action potentials
}

\author{
G. RICHARDSON† \\ School of Mathematics, University of Southampton, Southampton, SO17 1BJ, UK
}

[Received on 3 April 2008; revised on 9 July 2008; accepted on 25 November 2008]

\begin{abstract}
By application of matched asymptotic expansions, a simplified partial differential equation (PDE) model for the dynamic electrochemical processes occurring in the vicinity of a membrane, as ions selectively permeate across it, is formally derived from the Poisson-Nernst-Planck equations of electrochemistry. It is demonstrated that this simplified model reduces itself, in the limit of a long thin axon, to the cable equation used by Hodgkin and Huxley to describe the propagation of action potentials in the unmyelinated squid giant axon. The asymptotic reduction from the simplified PDE model to the cable equation leads to insights that are not otherwise apparent; these include an explanation of why the squid giant axon attains a diameter in the region of $1 \mathrm{~mm}$. The simplified PDE model has more general application than the Hodgkin-Huxley cable equation and can, e.g. be used to describe action potential propagation in myelinated axons and neuronal cell bodies.
\end{abstract}

Keywords: action potential; matched asymptotic expansions; electrolyte; Hodgkin-Huxley model; Poisson-Nernst-Planck equations.

\section{Introduction}

In most living cells, there is a potential difference between the two sides of the cell membrane-termed the transmembrane potential. This arises as a result of several factors. The most important of these being (i) charges on the lipids, which constitute the cell membrane, and (ii) a net charge in the electrolytic solutions in the immediate vicinity of the cell membrane. It is now known that the latter occurs due to the different compositions of the electrolytes on either side of the membrane and due to the presence of ion channels in the membrane. These ion channels are protein channels that straddle the cell membrane and allow for selective permeation of ions through it; there are, e.g. potassium channels that only allow potassium ions to pass through them. The origin of the transmembrane potential is illustrated in Fig. 1. Here, open potassium channels allow a flux of potassium ions across the membrane, from a region of high concentration in the intracellular space (bottom of the figure) to a region of low concentration in the extracellular space (top of the figure). As potassium ions cross the membrane, they carry charge with them, causing an increase in the magnitude of the potential difference between the two sides of the membrane (the transmembrane potential). Eventually, when the transmembrane potential becomes large enough, the electric force on the potassium ions becomes sufficient to counteract their concentration gradient and their transmembrane flux ceases.

\footnotetext{
$\dagger$ Email: G.Richardson@soton.ac.uk
} 


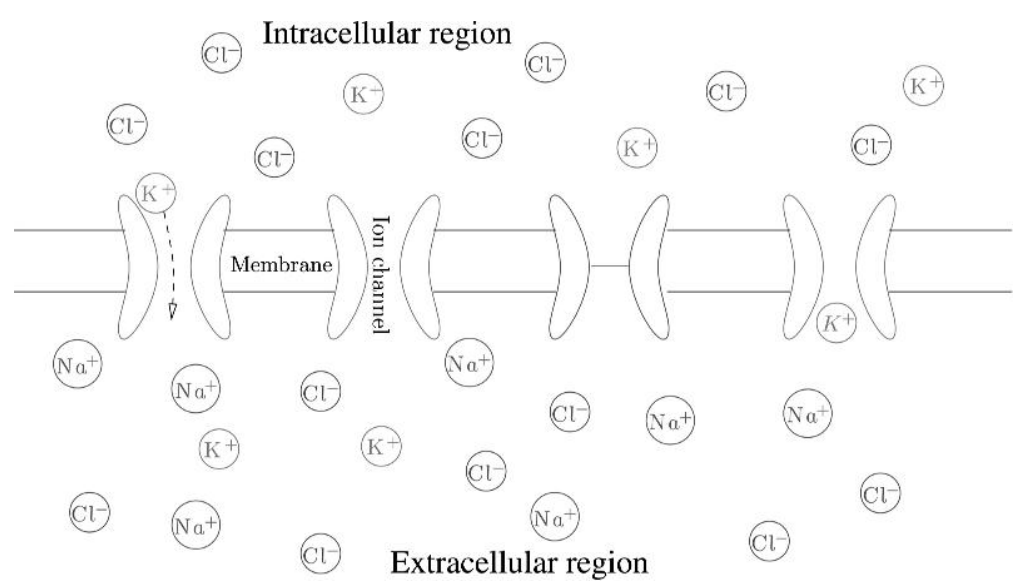

FIG. 1. A schematic of the membrane and how the membrane potential arises. Polarization of the membrane through the opening of potassium channels.

Action potentials are oscillations in the transmembrane potential, which typically occur over the order of milliseconds. In a wide range of cell types, such action potentials are observed to propagate as a wave, along the cell and (sometimes) between cells. Examples of propagating action potentials are found in neurons (e.g. Hodgkin \& Huxley, 1952d), where their purpose was to transmit signals between various parts of the organism, in cardiac myocytes (e.g. Luo \& Rudy, 1994) where they are responsible for the rhythmic contraction of the heart and in cells in the plant phloem (e.g. Mwesgwa et al., 1999; Fromm \& Bauer, 1994).

The first thorough investigation to be conducted on action potentials and their propagation was performed by Hodgkin and Huxley on the squid giant axon (Hodgkin et al., 1952; Hodgkin \& Huxley, 1952a,b,c). This was subsequently followed by a phenomenological, and extremely successful, model of action potential propagation in the squid giant axon (Hodgkin \& Huxley, 1952d). This model and modifications to it are still widely used to describe the electrical response of axons (see, e.g. Meunier \& Segev, 2002). In this context, we remark that the other extremely widely used model of neuron behaviour is a simple lumped parameter model of the neuron called the 'integrate-and-fire model' (see, e.g. Burkitt, 2006).

The basic physical processes behind the action potential are electrochemical in nature involving the migration of ions in vicinity of the membrane and through ion channels. In the context of the latter, we remark that because of their diameter, which is comparable to the diameter of a single ion, the ion flux through an ion channel cannot be quantitatively modelled using a simple continuum approach. We therefore adopt a phenomenological approach to modelling ion channel behaviour, considering them as devices that behave in a certain fashion in response to external stimuli (such as the transmembrane potential). At a mesoscopic level (on length scales of the order of nanometres), it is appropriate to describe the behaviour of the electrolytic solutions lying in the vicinity of the membrane with the timedependent, non-linear Poisson-Nernst-Planck (PNP) equations of electrochemistry (see, e.g. Newman $\&$ Thomas-Alyea, 2004). These equations predict approximate charge neutrality within a solution at a sufficient distance from surfaces. If a surface is charged, or associated with a charge, then a narrow Debye layer, with typical width of a few nanometres, results in the immediate vicinity of the membrane in which the electrolytic solution is highly charged and the concentration of negative ions does not even 
approximately balance that of positive ones. In equilibrium, the electric potential and ion concentrations in the Debye layer are predicted by the so-called 'Gouy-Chapman solution' (Gouy, 1910; Chapman, 1913) to the PNP equations. Given the vast differences in length scales between that of the Debye layer and other length scales encountered in problems as varied as modelling the electrical behaviour of an electrochemical cell, or that of a cell membrane, it is surprising how infrequently matched asymptotic expansions have been applied. Recent examples of the application of these techniques to electrochemical cells include Bazant et al. (2004, 2005) and Richardson \& King (2007). In the latter, a systematic analysis of the PNP equations is conducted in order to demonstrate the origin of the phenomenological Butler-Volmer equation; this arises as a boundary condition, at an electrode, on a diffusion equation that is used to model the behaviour of an electrolyte in the charge neutral region of an electrochemical cell.

Motivated by a desire to understand how ion channel behaviour on the microscale relates to the propagation of action potentials on the macroscale, we apply the methods of matched asymptotic analysis to a detailed model, based on the PNP equations, which describes the behaviour of electrolytic solutions lying on either side of a membrane through which ions permeate selectively via a system of ion channels. This analysis leads to a simplified (macroscale) model of the process and is as a result of finding a leading order, analytic, Debye layer solution in the immediate vicinity of the membrane and matching this to a reduced set of equations describing the behaviour of the electrolyte away from the Debye layers. We remark that the model we derive is similar to a model written down by Lindsay et al. (2004a), on the basis of their physical intuition. In addition, we consider how our model can be used to derive the cable equations of Hodgkin \& Huxley (1952d), for a sufficiently thin cylindrical axon via a singular perturbation analysis. Once again we note the work of Lindsay et al. (2004a), which investigates the links between the intuitive model they wrote down in the former part of their paper and the cable equation for a dendrite subject to external excitation. We note, however, that their work does not apply to the case of an axon transmitting an action potential, and indeed the asymptotic structure of the solution they use to derive the dendrite cable equation is quite different to that required to derive the cable equation in the context of an action potential transmitted along an axon.

The outline of this work is as follows. In Section 2, we formulate a model for the electrochemical behaviour of the electrolyte in the vicinity of the membrane. In Section 3, we use matched asymptotic expansions to derive a simplified model of the electrolyte, in which the membrane and surrounding Debye layers are reduced to a surface of infinitesimal thickness. This (simplified) model, which is the main result of our work, is stated in Section 4. In Section 5, we use this model to show how, by use of a singular perturbation analysis in the case of a sufficiently thin axon, the transmembrane potential obeys the 1D Hodgkin-Huxley cable equation. Finally, in Section 6, we draw our conclusions.

\section{An advection diffusion model for ion concentration in a dilute electrolytic solution}

Here, we formulate a model based on a three-species electrolyte, consisting of sodium, potassium and chloride ions. While there are many other ion species within the intracellular spaces, these three species are the most abundant.

\subsection{The geometry of the problem}

We aim to derive a simplified model of the electrochemical behaviour in the vicinity of a cell membrane for membrane geometries with radius of curvature much greater than the Debye length scale of the adjacent electrolytic solutions. Since the latter is typically very small, this assumption is not very 
restrictive. However, in order to simplify the presentation of the derivation of this simplified model, we shall perform its derivation under the assumption that the membrane is planar, occupying the region $\left|x^{*}\right|<\alpha$, as illustrated in Fig. 2 (this avoids unnecessary complications that arise on the introduction of curvilinear coordinates). Although the results derived in this fashion may appear to lack generality, it turns out that geometrical effects arising from membrane curvature leave the simplified model, which is a leading order asymptotic approximation, unaffected.

\subsection{The PNP equations in the electrolyte}

The PNP equations hold in the electrolyte on either side of the membrane. In the scenario where this is composed of the three species $\mathrm{Na}^{+}, \mathrm{K}^{+}$and $\mathrm{Cl}^{-}$, with concentrations $N, K$ and $C$, respectively, these can be written in the following form:

$$
\begin{gathered}
\frac{\partial N^{*}}{\partial t^{*}}+\nabla^{*} \cdot \mathcal{F}_{n}^{*}=0, \quad \text { where } \mathcal{F}_{n}^{*}=-D_{n}^{*}\left(\nabla N^{*}+\frac{F}{R T} N^{*} \nabla \phi^{*}\right), \\
\frac{\partial K^{*}}{\partial t^{*}}+\nabla^{*} \cdot \mathcal{F}_{k}^{*}=0, \quad \text { where } \mathcal{F}_{k}^{*}=-D_{k}^{*}\left(\nabla K^{*}+\frac{F}{R T} K^{*} \nabla \phi^{*}\right), \\
\frac{\partial C^{*}}{\partial t^{*}}+\nabla^{*} \cdot \mathcal{F}_{c}^{*}=0, \quad \text { where } \mathcal{F}_{c}^{*}=-D_{c}^{*}\left(\nabla C^{*}-\frac{F}{R T} C^{*} \nabla \phi^{*}\right), \\
\nabla^{*} \cdot\left(\varepsilon^{*} \nabla^{*} \phi^{*}\right)=F\left(C^{*}-N^{*}-K^{*}\right),
\end{gathered}
$$

in $\left|x^{*}\right|>\alpha$. Here, * s denotes dimensional variables; $F$ is Faraday's constant; $R$ is the universal gas constant; $T$ is the absolute temperature of the electrolyte; $\varepsilon^{*}$ is the permittivity of the electrolyte; $\phi^{*}$ is the potential; $\mathcal{F}_{n}^{*}, \mathcal{F}_{k}^{*}$ and $\mathcal{F}_{c}^{*}$ are the fluxes of sodium, potassium and chloride ions, respectively; $D_{n}^{*}, D_{k}^{*}$ and $D_{c}^{*}$ are the diffusivities of sodium, potassium and chloride ions, respectively; and $N, K$ and $C$ are the concentrations of sodium, potassium and chloride ions, respectively. These equations hold in the electrolyte on either side of the membrane. Here, the first parts of (1-3) represent conservation of the three species (sodium ions, potassium ions and chloride ions, respectively), while the second parts define their fluxes. Finally, (4) is Poisson's equation for the electric potential in the electrolyte.

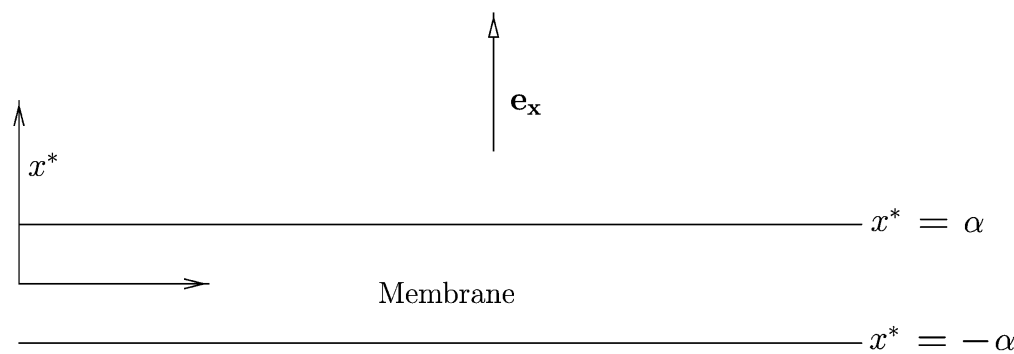

FIG. 2. The coordinate system around the membrane. 


\subsection{Poisson's equation in the membrane}

In the membrane itself, the electric potential satisfies Poisson's equation. Furthermore, we assume that a surface charge density $f^{*}$ lies on the inner leaflet of the membrane at $x^{*}=\alpha$ so that

$$
\nabla^{*} \cdot\left(\varepsilon^{*} \nabla^{*} \phi^{*}\right)=-f^{*} \Delta\left(x^{*}-\alpha\right) \quad \text { in } \quad-\alpha \leqslant x^{*} \leqslant \alpha,
$$

where $\Delta(\cdot)$ is the Dirac delta function.

\subsection{Boundary and jump conditions on the edge of the membrane}

The driving force for changes in the potential and electrochemical make up of the electrolytes adjacent to the membrane are transmembrane ion fluxes occurring through ion channels situated in the membrane. Here, we did not consider how this flux is maintained and, in particular, how it is related to the potential difference and concentration gradients across the membrane but remark that there are many models for these fluxes starting with those from the originalHodgkin-Huxley model (Hodgkin \& Huxley, 1952d). In order to keep the analysis general, we thus consider the flux of sodium ions, potassium ions and chloride ions in the positive $x$-direction across the membrane to be given by the functions $g_{n}^{*}, g_{k}^{*}$ and $g_{c}^{*}$, respectively (which would typically be chosen to fit experimental data). This gives rise to the following boundary conditions on $\mathcal{F}_{n}, \mathcal{F}_{k}$ and $\mathcal{F}_{c}$ :

$$
\begin{array}{llll}
\mathcal{F}_{n}^{*} \cdot \boldsymbol{e}_{x}=g_{n}^{*}, & \mathcal{F}_{k}^{*} \cdot \boldsymbol{e}_{x}=g_{k}^{*}, & \mathcal{F}_{c}^{*} \cdot \boldsymbol{e}_{x}=g_{c}^{*}, & \text { on } \quad x^{*}=\alpha, \\
\mathcal{F}_{n}^{*} \cdot \boldsymbol{e}_{x}=g_{n}^{*}, & \mathcal{F}_{k}^{*} \cdot \boldsymbol{e}_{x}=g_{k}^{*}, & \mathcal{F}_{c}^{*} \cdot \boldsymbol{e}_{x}=g_{c}^{*}, & \text { on } \quad x^{*}=-\alpha .
\end{array}
$$

In addition, we impose the standard jump conditions on the potential on the edge of the membrane

$$
\begin{array}{r}
{\left[\phi^{*}\right]_{x^{*}=\alpha^{-}}^{x^{*}=\alpha^{+}}=0, \quad\left[\varepsilon^{*} \frac{\partial \phi^{*}}{\partial x^{*}}\right]_{x^{*}=\alpha^{-}}^{x^{*}=\alpha^{+}}=0,} \\
{\left[\phi^{*}\right]_{x^{*}=-\alpha^{-}}^{x^{*}=-\alpha^{+}}=0, \quad\left[\varepsilon^{*} \frac{\partial \phi^{*}}{\partial x^{*}}\right]_{x^{*}=-\alpha^{-}}^{x^{*}=-\alpha^{+}}=0 .}
\end{array}
$$

REMARK. We should perhaps have considered the flux of ions from individual ion channels, rather than that from a continuum of channels as in (6-7), as these channels are typically spaced at distances comparable to, or larger than, the Debye length (the fundamental length scale of (1-4)). In this context, we note the work of Neuss-Radu \& Jäger (2005) in which a rigorous asymptotic derivation of boundary conditions of this form is made, by using homogenization methods on a periodic array of ion channels in the limit as membrane thickness tends to zero. Furthermore, we note that in the context of the matched asymptotic analysis we are about to perform, whether we use a boundary condition for a continuum of ion channels, like (6-7), or for a discrete array of channels spaced on the Debye length scale makes no difference as ion flux in the Debye layer makes only a small correction to the leading order solution at this scale.

\subsection{Current density}

The current density $j^{*}$ can be evaluated in terms of the difference in the fluxes of the positive and negative ions so that, in this case, we have

$$
j^{*}=F\left(\mathcal{F}_{n}^{*}+\mathcal{F}_{k}^{*}-\mathcal{F}_{c}^{*}\right)
$$




\subsection{Non-dimensionalization of the model}

We non-dimensionalize the Models (1-9) as follows:

$$
\begin{aligned}
& t^{*}=\tau t, \quad N^{*}=\Pi_{0} N, \quad K^{*}=\Pi_{0} K, \quad C^{*}=\Pi_{0} C, \\
& \phi^{*}=\frac{R T}{F} \phi, \quad g_{n}^{*}=G g_{n}, \quad g_{k}^{*}=G g_{k}, \quad g_{c}^{*}=G g_{c}, \quad f^{*}=\frac{R T \bar{\varepsilon}}{F L \delta} f, \\
& \boldsymbol{x}^{*}=L \boldsymbol{x}, \quad D_{n}^{*}=\bar{D} D_{n}, \quad D_{k}^{*}=\bar{D} D_{k}, \quad D_{c}^{*}=\bar{D} D_{c}, \\
& \varepsilon^{*}=\bar{\varepsilon} \varepsilon, \quad \mathcal{F}_{n}^{*}=G \mathcal{F}_{n}, \quad \mathcal{F}_{k}^{*}=G \mathcal{F}_{k}, \quad \mathcal{F}_{c}^{*}=G \mathcal{F}_{c}, \quad j^{*}=F G j .
\end{aligned}
$$

Here, $\Pi_{0}$ represents a typical ion concentration, $\tau$ is the typical timescale, $G$ is the typical transmembrane flux, $\bar{D}$ is a typical ion diffusivity, $\bar{\varepsilon}$ is a typical permittivity (e.g. that of the electrolyte) and $\delta$ is a dimensionless parameter, which is defined in (21). The potential $\phi^{*}$ has been non-dimensionalized with the thermal voltage $R T / F \approx 0.025 \mathrm{~V}$, in the usual manner (it is notable that the resting transmembrane potential is typically about $-3 R T / F$ ). On making this non-dimensionalization (and on choosing $L$ and $\tau$ as below), the governing equations and boundary conditions (1-9) reduce to the dimensionless form

$$
\begin{gathered}
\frac{\partial N}{\partial t}+\delta \nabla \cdot \mathcal{F}_{n}=0, \quad \text { where } \quad \mathcal{F}_{n}=-D_{n}(\nabla N+N \nabla \phi), \\
\frac{\partial K}{\partial t}+\delta \nabla \cdot \mathcal{F}_{k}=0, \quad \text { where } \quad \mathcal{F}_{k}=-D_{k}(\nabla K+K \nabla \phi), \\
\frac{\partial C}{\partial t}+\delta \nabla \cdot \mathcal{F}_{c}=0, \quad \text { where } \quad \mathcal{F}_{c}=-D_{c}(\nabla C-C \nabla \phi), \\
\nabla \cdot(\varepsilon \nabla \phi)=\frac{1}{\delta^{2}}(C-N-K),
\end{gathered}
$$

in $|x|>a$, which couples, via the jump and boundary conditions

$$
\begin{aligned}
& \mathcal{F}_{n} \cdot \boldsymbol{e}_{x}=g_{n}, \quad \mathcal{F}_{k} \cdot \boldsymbol{e}_{x}=g_{k}, \quad \mathcal{F}_{c} \cdot \boldsymbol{e}_{x}=g_{c}, \quad \text { on } \quad x=\delta a, \\
& {[\phi]_{x=\delta a^{-}}^{x=\delta a^{+}}=0, \quad\left[\varepsilon \frac{\partial \phi}{\partial x}\right]_{x=\delta a^{-}}^{x=\delta a^{+}}=0,} \\
& \mathcal{F}_{n} \cdot \boldsymbol{e}_{x}=g_{n}, \quad \mathcal{F}_{k} \cdot \boldsymbol{e}_{x}=g_{k}, \quad \mathcal{F}_{c} \cdot \boldsymbol{e}_{x}=g_{c}, \quad \text { on } \quad x=-\delta a, \\
& {[\phi]_{x=-\delta a^{-}}^{x=-\delta a^{+}}=0, \quad\left[\varepsilon \frac{\partial \phi}{\partial x}\right]_{x=-\delta a^{-}}^{x=-\delta a^{+}}=0,}
\end{aligned}
$$

to

$$
\nabla \cdot(\varepsilon \nabla \phi)=-\frac{f}{\delta} \Delta(x-\delta a) \quad \text { in } \quad-\delta a \leqslant x \leqslant \delta a .
$$

Here, the dimensionless parameters are defined by

$$
\delta=\frac{1}{L} \sqrt{\frac{\bar{\varepsilon} R T}{F^{2} \Pi_{0}}}, \quad a=\frac{\alpha}{\lambda_{D}} .
$$


and $L, \tau$ and $\lambda_{D}$ (the Debye length) are given by

$$
L=\frac{\bar{D} \Pi_{0}}{G}, \quad \tau=\frac{\Pi_{0}}{G} \lambda_{D}, \quad \lambda_{D}=\sqrt{\frac{\bar{\varepsilon} R T}{F^{2} \Pi_{0}}} .
$$

The length scale $L$ is chosen as the scale on which electric field contributions to the ion fluxes are of a comparable size to that of the transmembrane ion fluxes (i.e. of $O(G)$ ) and the timescale $\tau$ is that required to significantly change the potential across the Debye layers.

Henceforth, we take the dimensionless permittivity to be constant both within the electrolyte and the membrane. Assuming that we non-dimensionalize with the permittivity of the electrolyte, this gives

$$
\varepsilon=1 \text { for }|x|>\delta a, \quad \varepsilon=\varepsilon_{m} \text { for }|x|<\delta a,
$$

where $\varepsilon_{m}$ is the ratio of the membrane permittivity to that of the electrolyte. Finally, we note that the dimensionless current density is given by

$$
j=\mathcal{F}_{n}+\mathcal{F}_{k}-\mathcal{F}_{c} .
$$

\subsection{Size of parameters}

We base our parameter estimate on the experiments on squid giant axons conducted by Hodgkin \& Huxley (1952d). For the most part, these were conducted at a temperature of $T \approx 280 \mathrm{~K}$. We take the typical electrolyte concentration $\Pi_{0}=140 \mathrm{~mol} \mathrm{~m}^{-3}$ (corresponding to $140 \mathrm{mM}$ ). The diffusivity of $\mathrm{K}^{+}$ions is estimated as $\bar{D}=1.8 \times 10^{-9} \mathrm{~m}^{2} \mathrm{~s}^{-1}$ (the diffusivities of $\mathrm{Cl}^{-}$and $\mathrm{Na}^{+}$ions are of a similar size). The permittivity of the electrolyte (in the immediate vicinity of the membrane) we estimate as lying somewhere in the region $\bar{\varepsilon}=5-80 \varepsilon_{0}$ (i.e. $\bar{\varepsilon}=4.4 \times 10^{-11}$ to $7.1 \times 10^{-10} \mathrm{~A} \mathrm{~s}$ $\mathrm{V}^{-1} \mathrm{~m}^{-1}$ ). The universal gas constant and Faraday's constant are given by $R=8.31 \mathrm{~J} \mathrm{~mol}^{-1} \mathrm{~K}^{-1}$ and $F=9.65 \times 10^{4} \mathrm{~mol} \mathrm{~m}^{-2}$, respectively. We estimate the typical transmembrane ion conductances from data provided in Hodgkin \& Huxley (1952d) on $\mathrm{K}^{+}$conductances. Here, the electrical conductance of $\mathrm{K}^{+}$ions for a $50-\mathrm{mV}$ depolarization is (approximately) $130 \mathrm{~A} \mathrm{~V}^{-1} \mathrm{~m}^{-2}$, which corresponds to a conductance $G=6.7 \times 10^{-5} \mathrm{~mol} \mathrm{~m}^{-2} \mathrm{~s}^{-1}$, whereas for a $100-\mathrm{mV}$ depolarization, it is (approximately) $200 \mathrm{~A} \mathrm{~V}^{-1} \mathrm{~m}^{-2}$, which corresponds to a conductance $G=2.0 \times 10^{-4} \mathrm{~mol} \mathrm{~m}^{-2} \mathrm{~s}^{-1}$.

On the basis of the above-mentioned data, we estimate the Debye length as lying in the region $\lambda_{D}=$ $3.0 \times 10^{-10}$ to $1.1 \times 10^{-9} \mathrm{~m}$, the length scale of non-dimensionalization in the range $L=1.2 \times 10^{-3}$ to $3.7 \times 10^{-3} \mathrm{~m}$ and the timescale of non-dimensionalization as $\tau=2 \times 10^{-4}$ to $2 \times 10^{-3} \mathrm{~s}$. It is remarkable that length scale $L$ picked by this dimensional analysis is close to that of the radius of the larger squid giant axons, which have diameter in the region of $1 \mathrm{~mm}$ and that the timescale $\tau$ picked by this analysis corresponds closely to that for the action potential, which is the order of milliseconds.

\section{Derivation of a simplified model via a matched asymptotic analysis}

Here, we perform a boundary layer analysis in order to derive a simplified model describing the current flow in the vicinity of the membrane. As is typical in such electrochemical problems, it is necessary to introduce a narrow Debye layer in the immediate neighbourhood of the membrane surface in which charge neutrality is not even approximately satisfied (i.e. $C-N-K$ is not close to 0 ). In terms of (15), this region is defined by rescaling the $x$-coordinate (which lies perpendicular to the membrane) 
with $\delta$. The aim is then to match the solution in the Debye layer region to that in an outer region. We choose the length scale for this outer region to be $O(1)$, in dimensionless units (corresponding to something like $1 \mathrm{~mm}$ in dimensional units). This choice of outer length scale is the canonical one, and the simplified model we derive on this length scale can be applied to geometries with typical dimensions much smaller than it (e.g. vertebrate axons with a diameter of a few microns). The matching between the outer region and the Debye layer is not entirely straightforward and necessitates the introduction of a further, intermediate, region that has scale between that of the Debye layer and the outer. The various regions are illustrated in Fig. 3.

\subsection{The outer region in $x>0$}

We need to consider two outer regions - one on each side of the membrane. However, since the analysis is essentially the same in both regions, we limit our discussion to that in $x>0$, in which we denote variables by the superscript (out+), and that it will be helpful to think of as the intracellular space. Expanding variables as follows:

$$
\begin{aligned}
& N^{(\text {out }+)}=N_{0}^{(\text {out }+)}+\delta N_{1}^{(\text {out }+)}+\cdots, \quad K^{(\text {out }+)}=K_{0}^{(\text {out }+)}+\delta K_{1}^{(\text {out }+)}+\cdots, \\
& C^{(\text {out }+)}=C_{0}^{(\text {out }+)}+\delta C_{1}^{(\text {out }+)}+\cdots, \quad \phi^{(\text {out }+)}=\phi_{0}^{(\text {out }+)}+\delta \phi_{1}^{(\text {out }+)}+\cdots, \\
& \mathcal{F}_{n}^{\text {(out }+)}=\mathcal{F}_{n, 0}^{\text {(out }+)}+\cdots, \quad \mathcal{F}_{k}^{\text {(out }+)}=\mathcal{F}_{k, 0}^{\text {(out }+)}+\cdots, \quad \mathcal{F}_{c}^{\text {(out }+)}=\mathcal{F}_{c, 0}^{\text {(out }+)}+\cdots,
\end{aligned}
$$

and substituting into (12-15) and taking the leading terms gives

$$
\frac{\partial N_{0}^{(\text {out }+)}}{\partial t}=\frac{\partial K_{0}^{(\text {out }+)}}{\partial t}=\frac{\partial C_{0}^{(\text {out }+)}}{\partial t}=0, \quad C_{0}^{(\text {out }+)}-K_{0}^{(\text {out }+)}-N_{0}^{(\text {out }+)}=0,
$$

from which we deduce that

$$
N_{0}^{(\text {out }+)}=\bar{N}, \quad K_{0}^{(\text {out+) }}=\bar{K}, \quad C_{0}^{(\text {out }+)}=\bar{N}+\bar{K},
$$

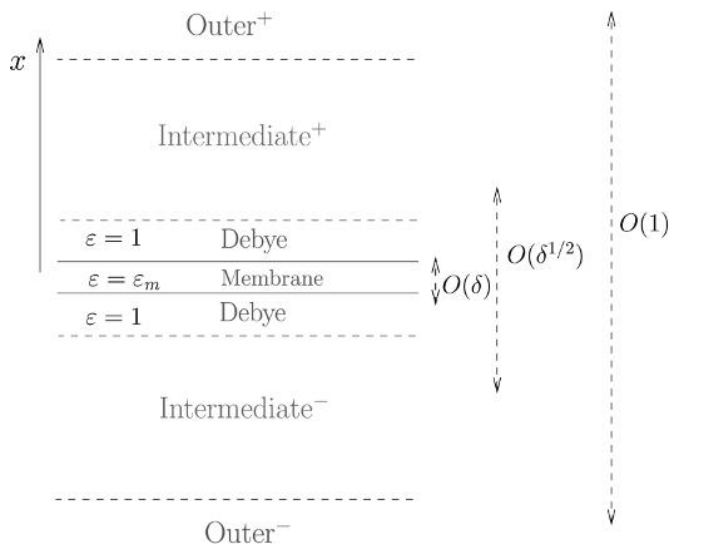

FIG. 3. Asymptotic regions of the problem. 
where $\bar{N}$ and $\bar{K}$ are constants. Proceeding to $O(\delta)$ in (12-15), we obtain

$$
\begin{aligned}
& \frac{\partial N_{1}^{(\text {out }+)}}{\partial t}+\nabla \cdot \mathcal{F}_{n, 0}^{\text {(out+) }}=0, \quad \mathcal{F}_{n, 0}^{\text {(out+) }}=-D_{n} \bar{N} \nabla \phi_{0}^{\text {(out }+)}, \\
& \frac{\partial K_{1}^{(\text {out }+)}}{\partial t}+\nabla \cdot \mathcal{F}_{k, 0}^{(\text {out }+)}=0, \quad \mathcal{F}_{k, 0}^{\text {(out }+)}=-D_{k} \bar{K} \nabla \phi_{0}^{(\text {out }+)}, \\
& \frac{\partial C_{1}^{(\text {out }+)}}{\partial t}+\nabla \cdot \mathcal{F}_{c, 0}^{\text {(out }+)}=0, \quad \mathcal{F}_{c, 0}^{(\text {out }+)}=D_{c}(\bar{N}+\bar{K}) \nabla \phi_{0}^{(\text {out }+)}, \\
& C_{1}^{(\text {out }+)}=N_{1}^{(\text {out }+)}+K_{1}^{(\text {out }+)} .
\end{aligned}
$$

Subtracting the third of these equations from the sum of the first two and using the fourth on the result leads to Laplace's equation for the leading order potential

$$
\nabla^{2} \phi_{0}^{(\text {out+) }}=0
$$

One quantity of interest is the leading order (dimensionless) current flux $\boldsymbol{j}_{0}^{\text {(out+) }}$, which is defined by

$$
j_{0}^{\text {(out+) }}=\mathcal{F}_{n, 0}^{\text {(out+) }}+\mathcal{F}_{k, 0}^{\text {(out }+)}-\mathcal{F}_{c, 0}^{\text {(out+) }} \Rightarrow j_{0}^{\text {(out+) }}=-\left(\left(D_{n}+D_{c}\right) \bar{N}+\left(D_{k}+D_{c}\right) \bar{K}\right) \nabla \phi_{0}^{\text {(out }+)} .
$$

Note that this is Ohm's law $\boldsymbol{j}=\sigma \boldsymbol{E}$ with effective (dimensionless) conductivity $\sigma^{+}=\left(\left(D_{n}+D_{c}\right) \bar{N}+\right.$ $\left.\left(D_{k}+D_{c}\right) \bar{K}\right)$.

\subsection{The outer region in $x<0$}

The analysis in the extracellular space $(x<0)$ mirrors that given above in the intracellular space $(x>0)$ with the one notable difference that the ion concentrations in $x<0$ are not necessarily the same as those in $x>0$ so that (22) and (23a-23b) are replaced by

$$
\begin{aligned}
N_{0}^{(\text {out }-)} & =\hat{N}, \quad K_{0}^{\text {(out-) }}=\hat{K}, \quad C_{0}^{\text {(out-) }}=\hat{N}+\hat{K}, \\
\mathcal{F}_{n, 0}^{\text {(out-) }} & =-D_{n} \hat{N} \nabla \phi_{0}^{\text {(out-) }}, \quad \mathcal{F}_{k, 0}^{\text {(out-) }}=-D_{k} \hat{K} \nabla \phi_{0}^{\text {(out-) }}, \quad \mathcal{F}_{c, 0}^{\text {(out-) }}=D_{c}(\hat{N}+\hat{K}) \nabla \phi_{0}^{\text {(out-) }}, \\
\nabla^{2} \phi_{0}^{\text {(out-) }} & =0 .
\end{aligned}
$$

The leading order (dimensionless) current flux $\boldsymbol{j}_{0}^{\text {(out-) }}$ is defined by

$$
j_{0}^{(\text {out }-)}=\mathcal{F}_{n, 0}^{(\text {out }-)}+\mathcal{F}_{k, 0}^{\text {(out-) }}-\mathcal{F}_{c, 0}^{\text {(out-) }} \Rightarrow j_{0}^{\text {(out-) }}=-\left(\left(D_{n}+D_{c}\right) \hat{N}+\left(D_{k}+D_{c}\right) \hat{K}\right) \nabla \phi_{0}^{(\text {out }-)},
$$

and the effective (dimensionless) conductivity by $\sigma^{-}=\left(\left(D_{n}+D_{c}\right) \hat{N}+\left(D_{k}+D_{c}\right) \hat{K}\right)$.

\subsection{The Debye layer}

Notably the leading order problems in the outer regions are singular perturbations to the full equations (12-15). This motivates the introduction of a boundary layer region, termed the Debye layer, in the 
vicinity of the membrane, which we obtain by rescaling distances normal to the membrane with the introduction of the new variable $\xi$ defined by

$$
x=\delta \xi
$$

In terms of this new variable, (12-15) for the region $|\xi|>a$ take the form

$$
\begin{aligned}
& \frac{\partial N^{(D)}}{\partial t}+\frac{\partial \mathcal{F}_{n x}^{(D)}}{\partial \xi}+\delta\left(\frac{\partial \mathcal{F}_{n y}^{(D)}}{\partial y}+\frac{\partial \mathcal{F}_{n z}^{(D)}}{\partial z}\right)=0, \quad \mathcal{F}_{n x}^{(D)}=-\frac{D_{n}}{\delta}\left(\frac{\partial N^{(D)}}{\partial \xi}+N^{(D)} \frac{\partial \phi^{(D)}}{\partial \xi}\right), \\
& \frac{\partial K^{(D)}}{\partial t}+\frac{\partial \mathcal{F}_{k x}^{(D)}}{\partial \xi}+\delta\left(\frac{\partial \mathcal{F}_{k y}^{(D)}}{\partial y}+\frac{\partial \mathcal{F}_{k z}^{(D)}}{\partial z}\right)=0, \quad \mathcal{F}_{k x}^{(D)}=-\frac{D_{k}}{\delta}\left(\frac{\partial K^{(D)}}{\partial \xi}+K^{(D)} \frac{\partial \phi^{(D)}}{\partial \xi}\right), \\
& \frac{\partial C^{(D)}}{\partial t}+\frac{\partial \mathcal{F}_{c x}^{(D)}}{\partial \xi}+\delta\left(\frac{\partial \mathcal{F}_{c y}^{(D)}}{\partial y}+\frac{\partial \mathcal{F}_{c z}^{(D)}}{\partial z}\right)=0, \quad \mathcal{F}_{c x}^{(D)}=-\frac{D_{c}}{\delta}\left(\frac{\partial C^{(D)}}{\partial \xi}-C^{(D)} \frac{\partial \phi^{(D)}}{\partial \xi}\right), \\
& \frac{\partial^{2} \phi^{(D)}}{\partial \xi^{2}}+\delta^{2}\left(\frac{\partial^{2} \phi^{(D)}}{\partial y^{2}}+\frac{\partial^{2} \phi^{(D)}}{\partial z^{2}}\right)=\left(C^{(D)}-N^{(D)}-K^{(D)}\right),
\end{aligned}
$$

and satisfy the boundary conditions on the normal fluxes

$$
\begin{aligned}
& \left.\mathcal{F}_{n x}^{(D)}\right|_{\xi=a}=g_{n},\left.\quad \mathcal{F}_{k x}^{(D)}\right|_{\xi=a}=g_{k},\left.\quad \mathcal{F}_{c x}^{(D)}\right|_{\xi=a}=g_{c}, \\
& \left.\mathcal{F}_{n x}^{(D)}\right|_{\xi=-a}=g_{n},\left.\quad \mathcal{F}_{k x}^{(D)}\right|_{\xi=-a}=g_{k},\left.\quad \mathcal{F}_{c x}^{(D)}\right|_{\xi=-a}=g_{c},
\end{aligned}
$$

on the edges of the membrane. In addition, they couple to a problem for the potential

$$
\frac{\partial^{2} \phi^{(D)}}{\partial \xi^{2}}+\delta^{2}\left(\frac{\partial^{2} \phi^{(D)}}{\partial y^{2}}+\frac{\partial^{2} \phi^{(D)}}{\partial z^{2}}\right)=0 \quad \text { in } \quad|\xi|>a
$$

via the jump conditions

$$
\begin{gathered}
\left.\frac{\partial \phi^{(D)}}{\partial \xi}\right|_{\xi=a^{+}}-\left.\varepsilon_{m} \frac{\partial \phi^{(D)}}{\partial \xi}\right|_{\xi=a^{-}}=-f,\left.\quad \phi^{(D)}\right|_{\xi=a^{-}}=\left.\phi^{(D)}\right|_{\xi=a^{+}}, \\
\left.\varepsilon_{m} \frac{\partial \phi^{(D)}}{\partial \xi}\right|_{\xi=-a^{+}}-\left.\frac{\partial \phi^{(D)}}{\partial \xi}\right|_{\xi=-a^{-}}=0,\left.\quad \phi^{(D)}\right|_{\xi=-a^{-}}=\left.\phi^{(D)}\right|_{\xi=-a^{+}} .
\end{gathered}
$$

Here, we have replaced the delta function in (20) by the inhomogeneous term on the right-hand side of (36a).

The Debye layer variables are expanded as follows:

$$
\begin{aligned}
& N^{(D)}=N_{0}^{(D)}+\delta N_{1}^{(D)}+\cdots, \quad K^{(D)}=K_{0}^{(D)}+\delta K_{1}^{(D)}+\cdots, \\
& C^{(D)}=C_{0}^{(D)}+\delta C_{1}^{(D)}+\cdots, \quad \phi^{(D)}=\phi_{0}^{(D)}+\delta \phi_{1}^{(D)}+\cdots, \\
& \mathcal{F}_{n}^{(D)}=\mathcal{F}_{n, 0}^{(D)}+\cdots, \quad \mathcal{F}_{k}^{(D)}=\mathcal{F}_{k, 0}^{(D)}+\cdots, \quad \mathcal{F}_{c}^{(D)}=\mathcal{F}_{c, 0}^{(D)}+\cdots
\end{aligned}
$$


3.3.1 Leading order solution. To leading order substitution of (37) into (29-32) gives

$$
\begin{gathered}
\frac{\partial N_{0}^{(D)}}{\partial \xi}+N_{0}^{(D)} \frac{\partial \phi_{0}^{(D)}}{\partial \xi}=0, \quad \frac{\partial K_{0}^{(D)}}{\partial \xi}+K_{0}^{(D)} \frac{\partial \phi_{0}^{(D)}}{\partial \xi}=0, \quad \frac{\partial C_{0}^{(D)}}{\partial \xi}-C_{0}^{(D)} \frac{\partial \phi_{0}^{(D)}}{\partial \xi}=0, \\
\frac{\partial^{2} \phi_{0}^{(D)}}{\partial \xi^{2}}=C_{0}^{(D)}-K_{0}^{(D)}-N_{0}^{(D)},
\end{gathered}
$$

which couple to

$$
\frac{\partial^{2} \phi_{0}^{(D)}}{\partial \xi^{2}}=0 \quad \text { in } \quad|\xi|<a
$$

via the jump conditions

$$
\begin{gathered}
\left.\frac{\partial \phi_{0}^{(D)}}{\partial \xi}\right|_{\xi=a^{+}}-\left.\varepsilon_{m} \frac{\partial \phi_{0}^{(D)}}{\partial \xi}\right|_{\xi=a^{-}}=-f,\left.\quad \phi_{0}^{(D)}\right|_{\xi=a^{-}}=\left.\phi_{0}^{(D)}\right|_{\xi=a^{+}}, \\
\left.\varepsilon_{m} \frac{\partial \phi_{0}^{(D)}}{\partial \xi}\right|_{\xi=-a^{+}}-\left.\frac{\partial \phi_{0}^{(D)}}{\partial \xi}\right|_{\xi=-a^{-}}=0,\left.\quad \phi_{0}^{(D)}\right|_{\xi=-a^{-}}=\left.\phi_{0}^{(D)}\right|_{\xi=-a^{+}},
\end{gathered}
$$

and satisfy the far-field conditions

$$
\begin{aligned}
& N_{0}^{(D)} \rightarrow \bar{N}, \quad K_{0}^{(D)} \rightarrow \bar{K}, \quad C_{0}^{(D)} \rightarrow \bar{N}+\bar{K} \quad \text { as } \quad \xi \rightarrow+\infty, \\
& N_{0}^{(D)} \rightarrow \hat{N}, \quad K_{0}^{(D)} \rightarrow \hat{K}, \quad C_{0}^{(D)} \rightarrow \hat{N}+\hat{K} \quad \text { as } \quad \xi \rightarrow-\infty,
\end{aligned}
$$

derived from matching to the outer solutions (22) as $\xi \rightarrow \infty$ and (28a-28c) as $\xi \rightarrow-\infty$.

We can integrate $(38 \mathrm{a}-38 \mathrm{c})$ in the standard fashion to give

$$
\left.\begin{array}{l}
N_{0}^{(D)}=\bar{N} \exp \left(V(y, z, t)-\phi_{0}^{(D)}\right) \\
K_{0}^{(D)}=\bar{K} \exp \left(V(y, z, t)-\phi_{0}^{(D)}\right) \\
C_{0}^{(D)}=(\bar{N}+\bar{K}) \exp \left(\phi_{0}^{(D)}-V(y, z, t)\right)
\end{array}\right\} \quad \text { in } \quad \xi>a,
$$

Here, in order to satisfy the far-field conditions (42-43), we have that

$$
\phi_{0}^{(D)} \rightarrow V(y, z, t) \quad \text { as } \quad \xi \rightarrow+\infty, \quad \phi_{0}^{(D)} \rightarrow W(y, z, t) \quad \text { as } \quad \xi \rightarrow-\infty,
$$

where $V$ and $W$ are, as yet, undetermined functions. Substitution of (44) and (45) into (39) leads to the Poisson-Boltzmann equations for the potential

$$
\begin{aligned}
& \phi_{0}^{(D)}{ }_{\xi \xi}=(\bar{N}+\bar{K})\left(\exp \left(\phi_{0}^{(D)}-V\right)-\exp \left(V-\phi_{0}^{(D)}\right)\right) \quad \text { in } \xi>a, \\
& \phi_{0}^{(D)}{ }_{\xi \xi}=(\hat{N}+\hat{K})\left(\exp \left(\phi_{0}^{(D)}-W\right)-\exp \left(W-\phi_{0}^{(D)}\right)\right) \quad \text { in } \quad \xi<-a .
\end{aligned}
$$


These equations couple to (40) via (41). Solving the resulting problem for the potential (i.e. (40), (41) and (46-47)) yields coupled Gouy-Chapman (Gouy, 1910; Chapman, 1913) type solutions in the electrolyte and a linear profile across the membrane (an illustrative plot is made in Fig. 4)

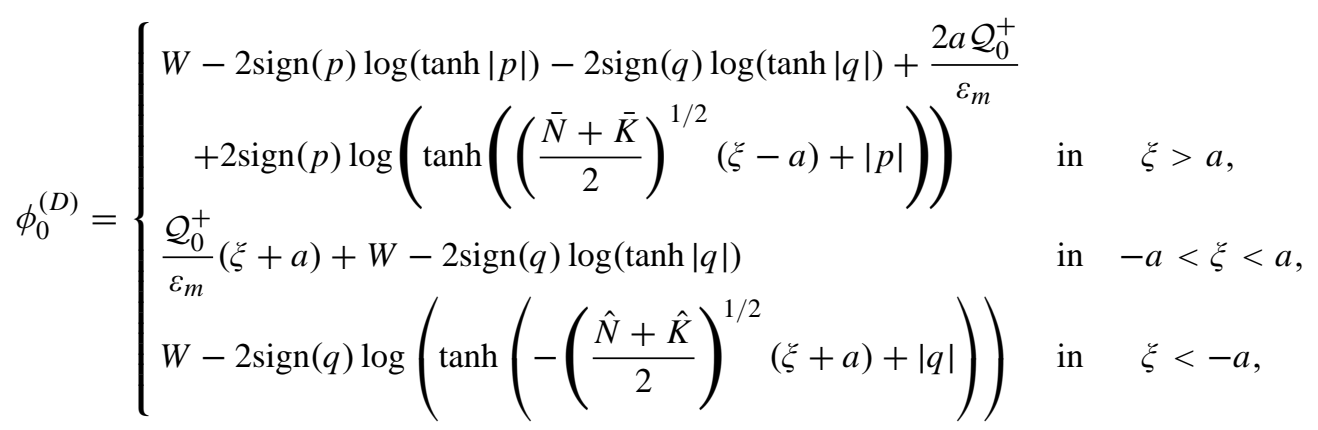

where

$$
p=\frac{1}{2} \operatorname{arcsinh}\left(\frac{2 \sqrt{2}(\bar{N}+\bar{K})^{1 / 2}}{\mathcal{Q}_{0}^{+}-f}\right), \quad q=\frac{1}{2} \operatorname{arcsinh}\left(\frac{2 \sqrt{2}(\hat{N}+\hat{K})^{1 / 2}}{\mathcal{Q}_{0}^{+}}\right) .
$$

Here, we have introduced the new variable $\mathcal{Q}_{0}^{+}$(at the expense of the variable $V$ ), which gives the dimensionless surface charge density per unit area of membrane in the Debye layer $\xi>a$. This and the corresponding surface charge density $\mathcal{Q}_{0}^{-}$in $x<-a$ are defined by

$$
\begin{aligned}
& \mathcal{Q}_{0}^{+}=f+\int_{a}^{\infty} N_{0}^{(D)}+K_{0}^{(D)}-C_{0}^{(D)} d \xi=f+\left.\frac{\partial \phi_{0}^{(D)}}{\partial \xi}\right|_{\xi=a^{+}}, \\
& \mathcal{Q}_{0}^{-}=\int_{-\infty}^{-a} N_{0}^{(D)}+K_{0}^{(D)}-C_{0}^{(D)} d \xi=\left.\frac{\partial \phi_{0}^{(D)}}{\partial \xi}\right|_{\xi=-a^{-}} .
\end{aligned}
$$
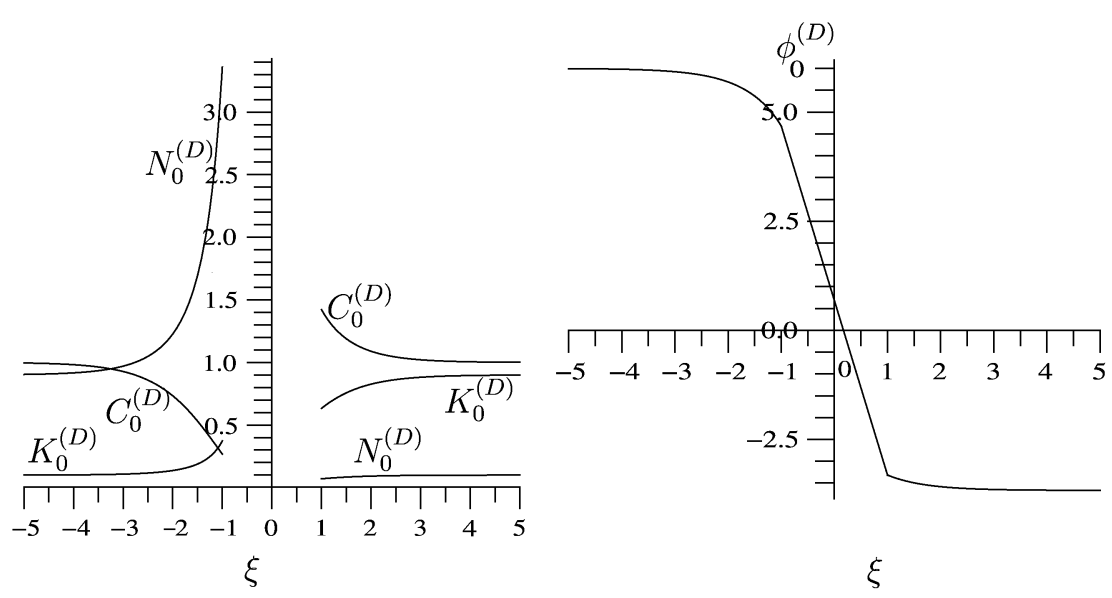

FIG. 4. Plots of (a) concentrations in the Debye layer region and (b) of the potential in the same region. Here, $f=-1.5$, $\mathcal{Q}_{0}^{+}=-2, a=1, \epsilon_{m}=0.5, W=6$ and for $\bar{N}+\bar{K}=\hat{N}+\hat{K}=1$. 
Integrating across the Debye layer, and noting that the derivative of the potential tends to zero at infinity, serves to show that $\mathcal{Q}_{0}^{-}=-\mathcal{Q}_{0}^{+}$, i.e. the charge in the left-hand Debye layer is equal to minus the charge in the right-hand Debye layer (where surface charge is included) and so in this sense the membrane can be seen to act as a capacitor. The potential $V$ at $\xi=+\infty$ is readily evaluated from (48) being given by

$$
V=W-2 \operatorname{sign}(p) \log (\tanh |p|)-2 \operatorname{sign}(q) \log (\tanh |q|)+\frac{2 a \mathcal{Q}_{0}^{+}}{\varepsilon_{m}} .
$$

In turn, this is easily rearranged to give a relationship between the leading order potential difference, $\Phi_{0}=V-W$, between $\xi=+\infty$ and $\xi=-\infty$ and the surface charge density $\mathcal{Q}_{0}^{+}$in $\xi>a$

$$
\Phi_{0}=\frac{2 a \mathcal{Q}_{0}^{+}}{\varepsilon_{m}}-2 \operatorname{sign}\left(p\left(\mathcal{Q}_{0}^{+}\right)\right) \log \left(\tanh \left|p\left(\mathcal{Q}_{0}^{+}\right)\right|\right)-2 \operatorname{sign}\left(q\left(\mathcal{Q}_{0}^{+}\right)\right) \log \left(\tanh \left|q\left(\mathcal{Q}_{0}^{+}\right)\right|\right)
$$

where $p$ and $q$ are defined in (49). This equation describes the capacitance of the membrane. In particular, it is noticeable that it comprises a linear part (the first term on the right-hand side) and a non-linear part. Thus, if $2 a / \varepsilon_{m} \gg 1$ (i.e. the membrane is sufficiently thick), the membrane behaves to a good approximation like a linear capacitor with capacitance $C \approx \varepsilon_{m} /(2 a)$. Note also that where the surface charge $f=0$, the function $\Phi_{0}$ is an even function of $\mathcal{Q}_{0}^{+}$. Illustrative plots of the capacitative properties of the membrane for a particular surface charge are shown in Fig. 5.

REMARK. It is important to state that the dielectric constant of the electrolyte is not now believed to remain constant throughout the electrolyte but in fact varies as a function of distance from any surface over a length scale of something like $10 \mathrm{~nm}$ (see e.g. Cherepanov et al., 2003), being depressed near the surface of the membrane and only achieving its bulk value $\approx 80 \varepsilon_{0}$ sufficiently far from the membrane. The Debye layer solution we have calculated on the assumption that the ratio of the permittivities between the membrane and the electrolyte is constant is not therefore expected to provide a quantitatively

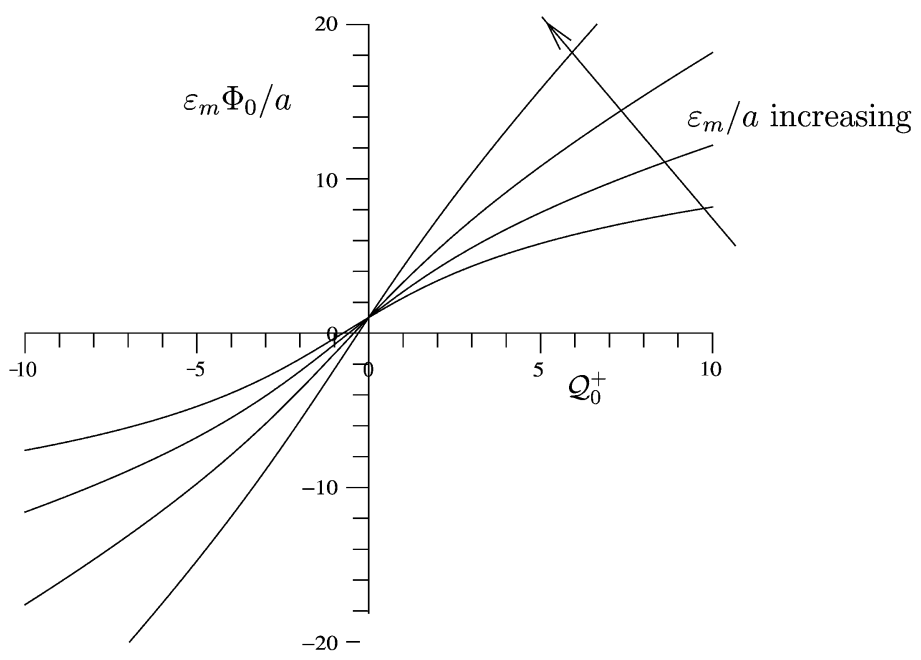

FIG. 5. The relationship between $\Phi_{0}$ and $\mathcal{Q}_{0}^{+}$for $\varepsilon_{m} / a=0,0.2,0.5,1$ in the case $f=-1.5$ and $\bar{N}+\bar{K}=\hat{N}+\hat{K}=1$. 
correct description. However, the qualitative description is reasonable, and in particular, this calculation shows that the important feature to come out of the leading order Debye layer analysis is a relationship between the surface charge density on the membrane and the potential drop across the Debye layer. In practice, this can always be measured experimentally. It is also notable that over the operating range of membrane potentials (up to about \pm 4 in these dimensionless units), the relationship between $\Phi_{0}$ and $\mathcal{Q}_{0}^{+}$is nearly always well approximated by a linear profile (see Fig. 5) of the form $\mathcal{Q}_{0}^{+}=C \Phi_{0}^{+}$.

3.3.2 First-order solution and solvability conditions. Proceeding to first order in the expansion (37) of (29-31) gives the following:

$$
\frac{\partial N_{0}^{(D)}}{\partial t}+\frac{\partial \mathcal{F}_{n x, 0}^{(D)}}{\partial \xi}=0, \quad \frac{\partial K_{0}^{(D)}}{\partial t}+\frac{\partial \mathcal{F}_{k x, 0}^{(D)}}{\partial \xi}=0, \quad \frac{\partial C_{0}^{(D)}}{\partial t}+\frac{\partial \mathcal{F}_{c x, 0}^{(D)}}{\partial \xi}=0 .
$$

Integrating between $\xi=a$ and $\xi=\infty$; substituting for $N_{0}^{(D)}, K_{0}^{(D)}$ and $C_{0}^{(D)}$ from (44) (in $\xi>a$ ); substituting for $\phi_{0}^{(D)}$ from (48) and (50) and applying the leading order flux conditions

$$
\left.\mathcal{F}_{n x, 0}^{(D)}\right|_{\xi=a}=g_{n},\left.\quad \mathcal{F}_{k x, 0}^{(D)}\right|_{\xi=a}=g_{k},\left.\quad \mathcal{F}_{c x, 0}^{(D)}\right|_{\xi=a}=g_{c}
$$

gives

$$
\begin{gathered}
\int_{a}^{\infty} \frac{\partial}{\partial t}\left(\frac{\bar{N}}{\tanh ^{2}\left(\left(\frac{\bar{N}+\bar{K}}{2}\right)^{1 / 2}(\xi-a)+p(y, z, t)\right)}\right) d \xi=g_{n}-\lim _{\xi \rightarrow+\infty} \mathcal{F}_{n x, 0}^{(D)} \\
\int_{a}^{\infty} \frac{\partial}{\partial t}\left(\frac{\bar{K}}{\tanh ^{2}\left(\left(\frac{\bar{N}+\bar{K}}{2}\right)^{1 / 2}(\xi-a)+p(y, z, t)\right)}\right) d \xi=g_{k}-\lim _{\xi \rightarrow+\infty} \mathcal{F}_{k x, 0}^{(D)} \\
\int_{a}^{\infty} \frac{\partial}{\partial t}\left((\bar{N}+\bar{K}) \tanh ^{2}\left(\left(\frac{\bar{N}+\bar{K}}{2}\right)^{1 / 2}(\xi-a)+p(y, z, t)\right)\right) d \xi=g_{c}-\lim _{\xi \rightarrow+\infty} \mathcal{F}_{c x, 0}^{(D)}
\end{gathered}
$$

Since the above-mentioned integrals diverge if either of $\bar{N}$ or $\bar{K}$ change with time, both are required to be static (over this timescale)-which is as one would expect from the outer solution. It is therefore only $p$ that varies with time, and each of these three equations are thus evolution equations for $p$, which is therefore overdetermined unless certain solvability conditions are satisfied. These are found by calculating the integrals given above, deriving expressions for $\partial p / \partial t$ and equating them equal. This yields two conditions on the ion fluxes, namely

$$
\frac{1}{\bar{N}}\left(g_{n}-\lim _{\xi \rightarrow+\infty} \mathcal{F}_{n x, 0}^{(D)}\right)=\frac{1}{\bar{K}}\left(g_{k}-\lim _{\xi \rightarrow+\infty} \mathcal{F}_{k x, 0}^{(D)}\right)=\frac{1}{(\bar{N}+\bar{K}) \tanh ^{2} p}\left(\lim _{\xi \rightarrow+\infty} \mathcal{F}_{c x, 0}^{(D)}-g_{c}\right) .
$$

Adding (52) to (53) and subtracting (54) provides a third relation (on noting that $\partial f / \partial t=0$ ), namely

$$
\frac{\partial}{\partial t} \int_{a}^{\infty} N_{0}^{(D)}+K_{0}^{(D)}-C_{0}^{(D)} d \xi=\frac{\partial \mathcal{Q}_{0}^{+}}{\partial t}=\left(g_{k}+g_{n}-g_{c}\right)-\lim _{\xi \rightarrow+\infty}\left(\mathcal{F}_{n x, 0}^{(D)}+\mathcal{F}_{k x, 0}^{(D)}-\mathcal{F}_{c x, 0}^{(D)}\right),
$$


which can be re-expressed as an equation for the conservation of charge in the Debye layer

$$
\begin{aligned}
& \frac{\partial \mathcal{Q}_{0}^{+}}{\partial t}=\left.j_{x, 0}^{(D)}\right|_{\xi=a}-\lim _{\xi \rightarrow+\infty} j_{x, 0}^{(D)} \quad, \text { where } \quad j_{x, 0}^{(D)}=\mathcal{F}_{n x, 0}^{(D)}+\mathcal{F}_{k x, 0}^{(D)}-\mathcal{F}_{c x, 0}^{(D)} \quad \text { and } \\
& \left.j_{x, 0}^{(D)}\right|_{\xi=a}=g_{k}+g_{n}-g_{c} .
\end{aligned}
$$

That is, the rate of change of surface charge density is equal to the current flux flowing into the Debye layer.

Analogous conditions may be derived on the left-hand side of the membrane in a similar fashion; they are

$$
\begin{aligned}
\frac{1}{\hat{N}}\left(g_{n}-\lim _{\xi \rightarrow-\infty} \mathcal{F}_{n x, 0}^{(D)}\right) & =\frac{1}{\hat{K}}\left(g_{k}-\lim _{\xi \rightarrow-\infty} \mathcal{F}_{k x, 0}^{(D)}\right)=\frac{1}{(\hat{N}+\hat{K}) \tanh ^{2} q}\left(\lim _{\xi \rightarrow-\infty} \mathcal{F}_{c x, 0}^{(D)}-g_{c}\right), \\
\frac{\partial \mathcal{Q}_{0}^{+}}{\partial t} & =\left(g_{k}+g_{n}-g_{c}\right)-\lim _{\xi \rightarrow-\infty}\left(\mathcal{F}_{n x, 0}^{(D)}+\mathcal{F}_{k x, 0}^{(D)}-\mathcal{F}_{c x, 0}^{(D)}\right) .
\end{aligned}
$$

The charge conservation condition in $\xi<-a$ (59) can be combined with that in $\xi>a$ (56) to eliminate $\partial \mathcal{Q}_{0}^{+} / \partial t$ and show that

$$
\lim _{\xi \rightarrow-\infty}\left(\mathcal{F}_{n x, 0}^{(D)}+\mathcal{F}_{k x, 0}^{(D)}-\mathcal{F}_{c x, 0}^{(D)}\right)=\lim _{\xi \rightarrow \infty}\left(\mathcal{F}_{n x, 0}^{(D)}+\mathcal{F}_{k x, 0}^{(D)}-\mathcal{F}_{c x, 0}^{(D)}\right), \quad \text { i.e. } \quad \lim _{\xi \rightarrow-\infty} j_{x, 0}^{(D)}=\lim _{\xi \rightarrow \infty} j_{x, 0}^{(D)},
$$

or in other words that the current density is continuous across the membrane to leading order.

The key conditions derived in this analysis, in terms of matching to regions outside the Debye layer, are (51), (55), (56), (58) and (60), which give the potential drop across the Debye layer, relations between the ion fluxes on the right-hand edge of the Debye layer, an evolution equation for the surface charge density in the Debye layer, relations for the ion fluxes on the left-hand edge of the Debye layer and continuity of current normal to the membrane across the Debye layer, respectively.

\subsection{Intermediate region}

It is notable that if we try to apply the conditions that we have just derived from the Debye layer analysis, namely (51), (55), (56), (58) and (60), to the outer problem as matching conditions on $x=0$, we end up with an overspecified problem. There is hence a need for two intermediate regions (on either side of the membrane), through which the Debye and outer regions match to each other. Since these regions perform an identical role, we look only at the one in $x>\delta a$; this we do by introducing the intermediate variable defined by

$$
x=\delta a+\delta^{1 / 2} w
$$

In terms of this new variable, (12-15) can be written as

$$
\frac{1}{\delta} \frac{\partial N^{(i+)}}{\partial t}+\frac{1}{\delta^{1 / 2}} \frac{\partial \mathcal{F}_{n x}^{(i+)}}{\partial w}+\frac{\partial \mathcal{F}_{n y}^{(i+)}}{\partial y}+\frac{\partial \mathcal{F}_{n z}^{(i+)}}{\partial z}=0, \mathcal{F}_{n x}^{(i+)}=-\frac{D_{n}}{\delta^{1 / 2}}\left(\frac{\partial N^{(i+)}}{\partial w}+N^{(i+)} \frac{\partial \phi^{(i+)}}{\partial w}\right)
$$




$$
\begin{gathered}
\frac{1}{\delta} \frac{\partial K^{(i+)}}{\partial t}+\frac{1}{\delta^{1 / 2}} \frac{\partial \mathcal{F}_{k x}^{(i+)}}{\partial w}+\frac{\partial \mathcal{F}_{k y}^{(i+)}}{\partial y}+\frac{\partial \mathcal{F}_{k z}^{(i+)}}{\partial z}=0, \quad \mathcal{F}_{k x}^{(i+)}=-\frac{D_{k}}{\delta^{1 / 2}}\left(\frac{\partial K^{(i+)}}{\partial w}+K^{(i+)} \frac{\partial \phi^{(i+)}}{\partial w}\right) \\
\frac{1}{\delta} \frac{\partial C^{(i+)}}{\partial t}+\frac{1}{\delta^{1 / 2}} \frac{\partial \mathcal{F}_{c x}^{(i+)}}{\partial w}+\frac{\partial \mathcal{F}_{c y}^{(i+)}}{\partial y}+\frac{\partial \mathcal{F}_{c z}^{(i+)}}{\partial z}=0, \quad \mathcal{F}_{c x}^{(i+)}=-\frac{D_{c}}{\delta^{1 / 2}}\left(\frac{\partial C^{(i+)}}{\partial w}-C^{(i+)} \frac{\partial \phi^{(i+)}}{\partial w}\right) \\
\delta \frac{\partial^{2} \phi^{(i+)}}{\partial w^{2}}+\delta^{2}\left(\frac{\partial^{2} \phi^{(i+)}}{\partial y^{2}}+\frac{\partial^{2} \phi^{(i+)}}{\partial z^{2}}\right)=\left(C^{(i+)}-N^{(i+)}-K^{(i+)}\right)
\end{gathered}
$$

The intermediate expansion proceeds as follows:

$$
\begin{aligned}
& N^{(i+)}=N_{0}^{(i+)}+\delta^{1 / 2} N_{1}^{(i+)}+\cdots, \quad K^{(i+)}=K_{0}^{(i+)}+\delta^{1 / 2} K_{1}^{(i+)}+\cdots \\
& C^{(i+)}=C_{0}^{(i+)}+\delta^{1 / 2} C_{1}^{(i+)}+\cdots, \quad \phi^{(i+)}=\phi_{0}^{(i+)}+\delta^{1 / 2} \phi_{1}^{(i+)}+\cdots \\
& \mathcal{F}_{n}^{(i+)}=\mathcal{F}_{n, 0}^{(i+)}+\cdots, \quad \mathcal{F}_{k}^{(i+)}=\mathcal{F}_{k, 0}^{(i+)}+\cdots, \quad \mathcal{F}_{c}^{(i+)}=\mathcal{F}_{c, 0}^{(i+)}+\cdots
\end{aligned}
$$

Substituting the above-mentioned expansion into (61b-63b) and (64) gives

$$
\begin{aligned}
& \frac{\partial N_{0}^{(i+)}}{\partial w}+N_{0}^{(i+)} \frac{\partial \phi_{0}^{(i+)}}{\partial w}=0, \quad \frac{\partial K_{0}^{(i+)}}{\partial w}+K_{0}^{(i+)} \frac{\partial \phi_{0}^{(i+)}}{\partial w}=0, \\
& \frac{\partial C_{0}^{(i+)}}{\partial w}-C_{0}^{(i+)} \frac{\partial \phi_{0}^{(i+)}}{\partial w}=0, \quad C_{0}^{(i+)}=N_{0}^{(i+)}+K_{0}^{(i+)}
\end{aligned}
$$

which in turn implies that $\phi_{0}^{(i+)}{ }_{w}=N_{0}^{(i+)}{ }_{w}=K_{0}^{(i+)}{ }_{w}=C_{0}^{(i+)}{ }_{w}=0$. Integrating and matching to the outer gives

$$
N_{0}^{(i+)}=\bar{N}, \quad K_{0}^{(i+)}=\bar{K}, \quad C_{0}^{(i+)}=\bar{N}+\bar{K}, \quad \phi_{0}^{(i+)}=\phi_{0}^{(i+)}(y, z, t) .
$$

Proceeding to first order in (64) and (61a-63a) gives

$$
\begin{array}{ll}
C_{1}^{(i+)}=N_{1}^{(i+)}+K_{1}^{(i+)}, & \frac{\partial N_{1}^{(i+)}}{\partial t}+\frac{\partial \mathcal{F}_{n x, 0}^{(i+)}}{\partial w}=0, \\
\frac{\partial K_{1}^{(i+)}}{\partial t}+\frac{\partial \mathcal{F}_{k x, 0}^{(i+)}}{\partial w}=0, & \frac{\partial C_{1}^{(i+)}}{\partial t}+\frac{\partial \mathcal{F}_{c x, 0}^{(i+)}}{\partial w}=0 .
\end{array}
$$

Adding the second of these equations to the third and subtracting the fourth and integrating the result with respect to $w$ yields (on using the fact $N_{1}^{(i+)}+K_{1}^{(i+)}-C_{1}^{(i+)}=0$ )

$$
\mathcal{F}_{n x, 0}^{(i+)}+\mathcal{F}_{k x, 0}^{(i+)}-\mathcal{F}_{c x, 0}^{(i+)}=j_{x, 0}^{(i+)}(y, z, t)
$$

(i.e. that the above-mentioned quantity is independent of $w$ ). 
The first-order fluxes normal to the membrane surface (found by substitution of (65) into $(61 b-63 b))$ are

$$
\begin{gathered}
\mathcal{F}_{n x, 0}^{(i+)}=-D_{n}\left(\frac{\partial N_{1}^{(i+)}}{\partial w}+\bar{N} \frac{\partial \phi_{1}^{(i+)}}{\partial w}\right), \quad \mathcal{F}_{k x, 0}^{(i+)}=-D_{k}\left(\frac{\partial K_{1}^{(i+)}}{\partial w}+\bar{K} \frac{\partial \phi_{1}^{(i+)}}{\partial w}\right), \\
\mathcal{F}_{c x, 0}^{(i+)}=-D_{c}\left(\frac{\partial N_{1}^{(i+)}}{\partial w}+\frac{\partial K_{1}^{(i+)}}{\partial w}-(\bar{N}+\bar{K}) \frac{\partial \phi_{1}^{(i+)}}{\partial w}\right) .
\end{gathered}
$$

Substitution of the above into (67c-67d) and elimination of $\partial \phi_{1}^{(i+)} / \partial w$ leads to a set of two coupled linear diffusion equations for $N_{1}^{(i+)}$ and $K_{1}^{(i+)}$

$$
\begin{aligned}
& \frac{\partial N_{1}^{(i+)}}{\partial t}=\frac{1}{\bar{K}\left(D_{k}+D_{c}\right)+\bar{N}\left(D_{n}+D_{c}\right)}\left[D_{k}\left(\bar{N}\left(D_{n}+D_{c}\right)+2 \bar{K} D_{c}\right) \frac{\partial^{2} K_{1}^{(i+)}}{\partial w^{2}}\right. \\
& \left.+\bar{K} D_{k}\left(D_{c}-D_{n}\right) \frac{\partial^{2} N_{1}^{(i+)}}{\partial w^{2}}\right] \\
& \frac{\partial K_{1}^{(i+)}}{\partial t}=\frac{1}{\bar{K}\left(D_{k}+D_{c}\right)+\bar{N}\left(D_{n}+D_{c}\right)}\left[D_{n}\left(\bar{K}\left(D_{k}+D_{c}\right)+2 \bar{N} D_{c}\right) \frac{\partial^{2} N_{1}^{(i+)}}{\partial w^{2}}\right. \\
& \left.+\bar{N} D_{n}\left(D_{c}-D_{k}\right) \frac{\partial^{2} K_{1}^{(i+)}}{\partial w^{2}}\right]
\end{aligned}
$$

while substitution of (69-70) into (68) leads, on differentiating the result with respect to $w$, to a secondorder equation for $\phi_{1}^{(i+)}$

$$
\left(\bar{N}\left(D_{c}-D_{s}\right)+\bar{K}\left(D_{c}-D_{k}\right)\right) \frac{\partial^{2} \phi_{1}^{(i+)}}{\partial w^{2}}=\left(D_{s}+D_{c}\right) \frac{\partial^{2} N_{1}^{(i+)}}{\partial w^{2}}+\left(D_{k}+D_{c}\right) \frac{\partial^{2} K_{1}^{(i+)}}{\partial w^{2}} .
$$

This equation can be solved for $\phi_{1}^{(i+)}$ once $N_{1}^{(i+)}$ and $K_{1}^{(i+)}$ have been determined from (71-72).

\subsection{Matching}

At this stage, the most important points to be taken from the intermediate analysis comes from (66) and (68), which essentially show that $N, K, C, \phi$ and $j_{x}=\mathcal{F}_{n x}+\mathcal{F}_{k x}-\mathcal{F}_{c x}$ are all constant across the intermediate layer to leading order (although not necessarily the individual fluxes $\mathcal{F}_{n x}, \mathcal{F}_{k x}$ and $\mathcal{F}_{c x}$ ). Matching these results from the intermediate to the (out+) outer and Debye layer regions on either side of it and thence to the (out-) region in $x<-\delta a$ via the $(i-)$ intermediate region give the results that

$$
\begin{aligned}
\left.\phi_{0}^{(\text {out }+)}\right|_{x=0^{+}}-\left.\phi_{0}^{(\text {out }-)}\right|_{x=0^{-}}= & \frac{2 a \mathcal{Q}_{0}^{+}}{\varepsilon_{m}}-2 \operatorname{sign}\left(p\left(\mathcal{Q}_{0}^{+}\right)\right) \log \left(\tanh \left|p\left(\mathcal{Q}_{0}^{+}\right)\right|\right) \\
& -2 \operatorname{sign}\left(q\left(\mathcal{Q}_{0}^{+}\right)\right) \log \left(\tanh \left|q\left(\mathcal{Q}_{0}^{+}\right)\right|\right) \\
\left.j_{x, 0}^{(\text {out }+)}\right|_{x=0^{+}}= & \left.j_{x, 0}^{\text {(out-) }}\right|_{x=0^{-}}, \quad \frac{\partial \mathcal{Q}_{0}^{+}}{\partial t}=\left(g_{k}+g_{n}-g_{c}\right)-\left.j_{x, 0}^{(\text {out }+)}\right|_{x=0^{+}}
\end{aligned}
$$


where $p(\cdot)$ and $q(\cdot)$ are defined in (49). In order to derive this result, we made use of the Debye layer conditions (51), (60) and (56). Thus, we see that in terms of the outer region, the normal current density crossing the membrane is continuous, while the surface charge density $\mathcal{Q}_{0}^{+}$evolves according to an equation that depends upon the flux of ions across the membrane and the current density in the outer region on the membrane. The potential drop across the membrane is then given in terms of $\mathcal{Q}_{0}^{+}$by the capacitance relation (74). This gives a well-posed outer problem when the ion fluxes $g_{n}, g_{k}$ and $g_{c}$ are specified.

In the next section, we give examples of the solution to this problem. However, before doing so, we refer the reader to Appendix A in which it is shown that the matching gives rise to the right number of boundary conditions on (71-73) for the first-order variables $N_{1}^{(i+)}, K_{1}^{(i+)}$ and $\phi_{1}^{(i+)}$ and furthermore provides a mechanism for adjusting the ion fluxes occurring on the outer edge of the Debye layer to those occurring on the inner edge of the outer region.

\section{The simplified PDE model}

We start by stating the simplified (outer) problem for a membrane with general geometry where the membrane is traversed by fluxes $g_{n}, g_{k}$ and $g_{c}$, of sodium, potassium and chloride ions, respectively. The matching analysis performed in Section 3 is unaffected (to the orders considered), provided the radius of curvature of the membrane is large with respect to the Debye length. Thus, the outer problem, which is illustrated in Fig. 6, takes the form

$$
\begin{gathered}
\nabla^{2} \phi=0 \quad \text { in } \quad \Omega \quad \text { and } \Omega^{c}, \\
\left.\sigma_{\text {in }} \frac{\partial \phi}{\partial n}\right|_{\partial \Omega}=\left.\sigma_{\text {out }} \frac{\partial \phi}{\partial n}\right|_{\partial \Omega^{c}}, \\
{[\phi]_{\partial \Omega^{c}}^{\partial \Omega}, \quad \mathcal{Q}=\mathcal{S}(\Phi),} \\
\frac{\partial \mathcal{Q}}{\partial t}=\left(g_{n}+g_{k}-g_{c}\right)-\left.\sigma_{\text {in }} \frac{\partial \phi}{\partial n}\right|_{\partial \Omega} .
\end{gathered}
$$

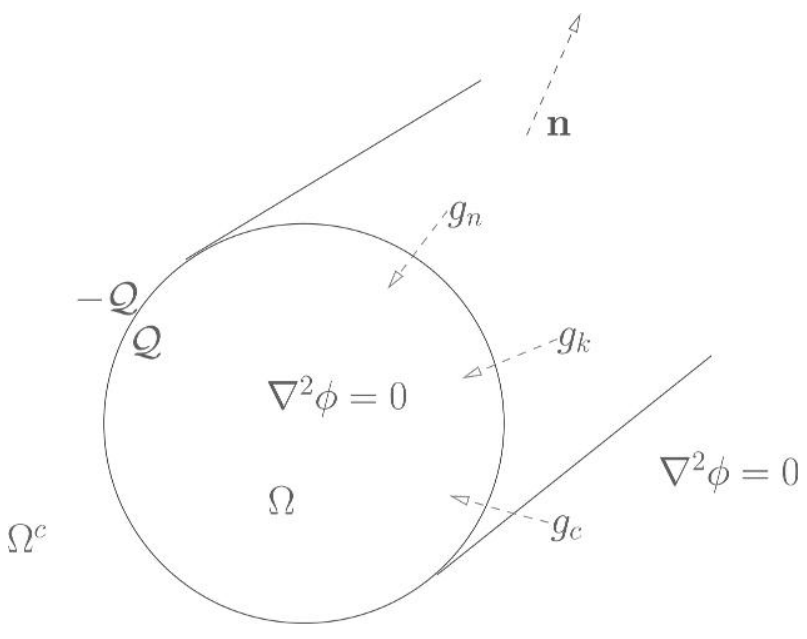

FIG. 6. The geometry of the model. 
Here, $\boldsymbol{n}$ is the outward normal to the region $\Omega, \sigma_{\text {in }}=\left(\left(D_{n}+D_{c}\right) \bar{N}+\left(D_{k}+D_{c}\right) \bar{K}\right), \sigma_{\text {out }}=\left(\left(D_{n}+\right.\right.$ $\left.\left.D_{c}\right) \hat{N}+\left(D_{k}+D_{c}\right) \hat{K}\right)$ and $\mathcal{S}$ is the non-linear capacitance of the membrane, i.e. it is the inverse of the function given in (74). However, as stated previously, we expect that in the ranges of membrane potential typically observed, it can, to a good approximation, be linearized to the form $\mathcal{S}(\Phi)=C \Phi$ (where the constant $C$ is the 'linear' capacitance of the membrane). The functions $g_{n}, g_{k}$ and $g_{c}$, which give the transmembrane fluxes, will typically be functions of the potential difference across the membrane as opposed to $\Phi$, which is the potential difference across the membrane and the Debye layers. However, given $\Phi$, it is possible to calculate the potential difference across the membrane from the explicit solution for the leading order Debye region potential (48).

In order to close the problem, it is generally necessary to pose a far-field condition in $\Omega^{c}$; typically, this might take the form

$$
\phi \rightarrow 0 \quad \text { as } \quad|x| \rightarrow+\infty
$$

\section{Derivation of the Hodgkin-Huxley cable equation for a thin unmyelinated axon}

Here, we consider a thin radially symmetric axon with dimensionless radius $\epsilon$ (where $\epsilon \ll 1$ ) suspended in a bath of electrolytic solution (this is the scenario considered by Hodgkin \& Huxley, 1952d) and is illustrated schematically in Fig. 6. We use cylindrical coordinates with the $z$-axis running along the centre of the axon and denote the position of the membrane by $r=\epsilon$. In order to obtain a solution to this problem, we will need to introduce an inner region lying with an $O(\epsilon)$ distance of the axon and an exterior region lying and $O\left(\epsilon^{1 / 2}\right)$ distance from the axon.

\subsection{Inner region}

In the inner region, we rescale coordinates as follows:

$$
z=\epsilon^{1 / 2} \zeta, \quad r=\epsilon R
$$

and denote variables by the superscript $(i)$. Furthermore, we shall assume axial symmetry (so that $\left.\phi^{(\mathrm{in})}=\phi^{(\mathrm{in})}(\zeta, R)\right)$. In terms of the new coordinates, (76-79) become

$$
\begin{aligned}
& \frac{1}{\epsilon R} \frac{\partial}{\partial R}\left(R \frac{\partial \phi^{(\mathrm{in})}}{\partial R}\right)+\frac{\partial^{2} \phi^{(\mathrm{in})}}{\partial \zeta^{2}}=0 \quad \text { for all } R, \\
& \left.\phi^{(\mathrm{in})}\right|_{R=1^{-}}-\left.\phi^{(\mathrm{in})}\right|_{R=1^{+}}=\Phi,\left.\quad \sigma^{-} \frac{\partial \phi^{(\mathrm{in})}}{\partial R}\right|_{R=1^{-}}=\left.\sigma^{+} \frac{\partial \phi^{(\mathrm{in})}}{\partial R}\right|_{R=1^{+}}, \\
& \mathcal{Q}=\mathcal{S}(\Phi), \quad \frac{\partial \mathcal{Q}}{\partial t}=\left(g_{n}+g_{k}-g_{c}\right)-\left.\sigma^{-} \frac{1}{\epsilon} \frac{\partial \phi^{(\mathrm{in})}}{\partial R}\right|_{R=1^{-}},
\end{aligned}
$$

where here $\sigma^{-}=\left(\left(D_{n}+D_{c}\right) \bar{N}+\left(D_{k}+D_{c}\right) \bar{K}\right)$ and $\sigma^{+}=\left(\left(D_{n}+D_{c}\right) \hat{N}+\left(D_{k}+D_{c}\right) \hat{K}\right)$. We look for an asymptotic solution to (81-83) of the form

$$
\begin{aligned}
\mathcal{Q} & =\mathcal{Q}_{0}+\epsilon \log \epsilon \mathcal{Q}_{1}+\epsilon \mathcal{Q}_{2}+\cdots, \quad \Phi=\Phi_{0}+\epsilon \log \epsilon \Phi_{1}+\epsilon \Phi_{2}+\cdots, \\
\phi^{(\mathrm{in})} & = \begin{cases}\Phi_{0}(\zeta, t)+\epsilon \log \epsilon\left(\phi_{1}^{(\mathrm{in})}(\zeta, t)+\Phi_{1}(\zeta, t)\right)+\epsilon\left(\phi_{2}^{(\mathrm{in})}(R, \zeta, t)+\Phi_{2}(\zeta, t)\right)+\cdots & 0 \leqslant R<1, \\
\epsilon \log \epsilon \phi_{1}^{(\mathrm{in})}(\zeta, t)+\epsilon \phi_{2}^{(\mathrm{in})}(R, \zeta, t)+\cdots & R>1 .\end{cases}
\end{aligned}
$$


Here, the presence of the $O(\epsilon \log \epsilon)$ terms is a result of the far-field logarithmic singularity encountered when solving Laplace's equation (81) in cylindrical coordinates; as, we shall show, this necessitates the introduction of an 'exterior' region far from the centre of the axon and it is the matching between this region and the present, inner, region, which gives rise to the logarithmic terms. Substitution of the above-mentioned expansion into (81-82) at $O(1)$ yields

$$
\begin{aligned}
& \frac{1}{R} \frac{\partial}{\partial R}\left(R \frac{\partial \phi_{2}^{(\mathrm{in})}}{\partial R}\right)=-\Phi_{0, \zeta \zeta} \quad \text { in } \quad R<1, \quad \frac{1}{R} \frac{\partial}{\partial R}\left(R \frac{\partial \phi_{2}^{(\mathrm{in})}}{\partial R}\right)=0 \quad \text { in } \quad R>1, \\
&\left.\phi_{2}^{(\mathrm{in})}\right|_{R=1^{-}}=\left.\phi_{2}^{(\mathrm{in})}\right|_{R=1^{+}},\left.\quad \sigma^{-} \frac{\partial \phi_{2}^{(\mathrm{in})}}{\partial R}\right|_{R=1^{-}}=\left.\sigma^{+} \frac{\partial \phi_{2}^{(\mathrm{in})}}{\partial R}\right|_{R=1^{+}},
\end{aligned}
$$

with solution

$$
\phi_{2}^{\text {(in) }}=\left\{\begin{array}{ll}
-\frac{\Phi_{0, \zeta \zeta}\left(R^{2}-1\right)}{4}+\gamma(\zeta, t) & \text { in } R<1 \\
-\frac{\Phi_{0, \zeta \zeta} \sigma^{-}}{2 \sigma^{+}} \log R+\gamma(\zeta, t) & \text { in } R>1
\end{array} .\right.
$$

\subsection{Exterior region}

The logarithmic divergence of the solution in the inner region as $R \rightarrow \infty$ signifies the existence of a further layer outside this inner. In order to investigate the solution here, we rescale coordinates as follows:

$$
z=\epsilon^{1 / 2} \zeta, \quad r=\epsilon^{1 / 2} \rho, \quad\left(\rho=\epsilon^{1 / 2} R\right)
$$

so that (76) now takes the form

$$
\frac{1}{\rho} \frac{\partial}{\partial \rho}\left(\rho \frac{\partial \phi^{(e)}}{\partial \rho}\right)+\frac{\partial^{2} \phi^{(e)}}{\partial \zeta^{2}}=0 .
$$

The expansion in this region proceeds as follows: $\phi^{(e)}=\epsilon \phi_{2}^{(e)}+\cdots$.

\subsection{Matching}

In order to match the inner region to the exterior, we require

$$
\phi_{1}^{(\mathrm{in})}=-\frac{\Phi_{0, \zeta \zeta} \sigma^{-}}{4 \sigma^{+}},
$$

so that we can write the far-field behaviour of the inner region in the form

$$
\phi^{(\text {in })} \sim-\epsilon \frac{\Phi_{0, \zeta \zeta} \sigma^{-}}{2 \sigma^{+}} \log \left(\epsilon^{1 / 2} R\right)+\epsilon \gamma(\zeta, t) \quad \text { as } \quad R \rightarrow+\infty .
$$

In turn, it follows that the matching condition on $\phi_{2}^{(e)}$ is

$$
\phi_{2}^{(e)} \sim-\frac{\Phi_{0, \zeta \zeta} \sigma^{-}}{2 \sigma^{+}} \log \rho+\gamma(\zeta, t) \quad \text { as } \quad \rho \rightarrow 0
$$




\subsection{Exterior region.}

The problem for the leading order exterior potential is thus

$$
\begin{gathered}
\frac{1}{\rho} \frac{\partial}{\partial \rho}\left(\rho \frac{\partial \phi_{2}^{(e)}}{\partial \rho}\right)+\frac{\partial^{2} \phi_{2}^{(e)}}{\partial \zeta^{2}}=0, \\
\phi_{2}^{(e)} \sim-\frac{\Phi_{0, \zeta \zeta} \sigma^{-}}{2 \sigma^{+}} \log \rho \quad \text { as } \quad \rho \rightarrow 0, \\
\phi_{2}^{(e)} \rightarrow 0 \quad \text { as } \quad \rho \rightarrow \infty,
\end{gathered}
$$

and its solution can be used to determine $\gamma(\zeta, t)$.

\subsection{Inner region and the cable equation}

Returning to the inner region, we use the correction to the inner solution that we have derived in (84) to determine the evolution of the leading order potential $\Phi$ inside the axon. Substituting (83a) into (83b) and using the solution calculated in (84) to determine leading order radial derivative of $\phi$ on $R=1^{-}$, we obtain the Hodgkin-Huxley cable equation

$$
\mathcal{S}^{\prime}(\Phi) \frac{\partial \Phi}{\partial t}=\sigma^{-} \frac{\partial^{2} \Phi}{\partial \zeta^{2}}+\left(g_{c}-g_{k}-g_{n}\right),
$$

where we have dropped sub- and superscripts.

REMARK 1. Recall that the axial coordinate $\zeta$ in (86) has been rescaled with $\epsilon^{1 / 2}$ (where $\epsilon$ measures the ratio of the radius of the axon to the length scale $L$ ). We consequently expect that the dimensional velocity $c$ of a propagating action potential scales with $\epsilon^{1 / 2}$ (i.e. thicker axons give rise to quicker waves). This is indeed what is observed (Hodgkin \& Huxley, 1952d). Physically this phenomenon is a consequence of the axial conductivity of the axon scaling with cross-sectional area while the capacitance, per unit length, and the number of ion channels, per unit length, scale with axon radius. An important corollary of this remark on the speed of action potential propagation is that increases in action potential velocity with (axon radius) ${ }^{1 / 2}$ only occur provided that (axon radius) $\ll L$; the small $\epsilon$ asymptotics breaks down after this point. Thus, when axon radius becomes comparable to $L$ (recall we estimated $L$ to be of $O(1 \mathrm{~mm})$ ), we cannot expect action potential velocity to increase as quickly with further increases in radius. From an evolutionary point of view, we expect squids to 'try' to maximize the speed of propagation, subject to certain costs, in order to escape from predators or catch prey. It is therefore not surprising that the squid giant axon has a diameter about $1 \mathrm{~mm}$ (i.e. of $O(L)$ ) as this is the size at which further increases in radius only give rise to small gains in action potential velocity.

REMARK 2. It is notable that the derivation presented above is very different to the derivation of the cable equation, in the context of a dendrite, presented by Lindsay et al. (2004a). In the latter, axial distance along the dendrite/axon is not rescaled with $\epsilon^{1 / 2}$ and the expansion of (inner) variables is conducted in powers of $\epsilon^{2}$ (i.e. $\varepsilon$ in the notation of Lindsay et al., 2004a) rather than in powers of $\epsilon$ and $\epsilon \log \epsilon$. 


\section{Conclusions}

We have begun by formulating a microscopic continuum model, based on the PNP equations of electrochemistry, for the electrical behaviour of two electrolytic solutions separated by a membrane through which ions permeate (selectively) via a system of ion channels. This model is capable of resolving features down to the thickness of the membrane. We then applied the method of matched asymptotic expansions to the microscopic model using, as our small parameter, the ratio of the Debye length to $L$, the length scale at which electrical contributions to the ion flux are comparable to the transmembrane ion fluxes through the ion channels. The resulting analysis leads to a simplified model (76-79), on the $O(L)$ length scale, for a charge neutral electrolytic solution in which charge is conducted via a version of Ohm's law (76). The membrane (and the Debye layers on either side of it) appear, at this length scale, as an infinitesimally thin interface that is able to store charge capacitative. Since there is no net charge on the interface, only a separation of charge across it, the normal current flowing into one side of the membrane is equal to the normal current flowing out the other side (77). Furthermore, charge conservation, which is embedded in the PNP equations, ensures that the rate of change of surface charge density in the Debye layer outside the membrane (this is balanced by an equal but opposite charge density on the inner side) is equal to the normal current flux flowing into the Debye layer from the electrolyte plus the current flux flowing into it through the ion channels (79). The model is closed by a non-linear relation between the potential drop across the membrane and the surface charge density stored in the Debye layers outside the membrane.

We remark further that although the derivation of the simplified model (76-79) suggests that it applies specifically to processes occurring on an $O(L)$ length scale, it has greater generality than that and can be applied to processes occurring on any length scale much greater than the Debye length. As an example of its application over a length scale smaller than $L$, we considered the transmission of an action potential in a thin (diameter $\ll L$ ), uniform, unmyelinated axon and showed that the simplified model (76-79) can itself be asymptotically approximated by the cable equations formulated in Hodgkin \& Huxley (1952d). Notably, we are able to use the Hodgkin and Huxley experimental data to estimate the critical axon radius, below which the cable equation description is appropriate, as about $1 \mathrm{~mm}$. Furthermore, since large squid giant axons have radius comparable to $1 \mathrm{~mm}$, we hypothesize that the solution to the cable equation may not give an entirely accurate description of action potential propagation in these structures; in this context, we note the work by Lindsay et al. (2004b), which points out systematic discrepancies between the wave speed predicted by the Hodgkin-Huxley model and that measured experimentally. In further work that we intend to carry out, numerical simulations of the travelling wave solutions to the simplified model (76-79) will be used to evaluate the action potential wave speed and, in turn, compared to the experimental data.

\section{Acknowledgement}

I thank Professor Stephen Coombes for a number of useful discussions and comments on this work.

\section{REFERENCES}

BAZANT, M. Z., ChU, K. T. \& BAYLY, B. J. (2005) Current voltage relations for electrochemical thin films. SIAM J. Appl. Math., 65, 1463-1484.

Bazant, M. Z., Thornton, K. \& Ajdari, A. (2004) Diffuse charge dynamics in electrochemical systems. Phys. Rev. E, 70, 0121506. 
BurkitT, A. N. (2006) A review of the integrate and fire neuron model: I. Homogeneous synaptic input. Biol. Cybern., 95, 1-19.

Chapman, D. L. (1913) A contribution to the theory of electrocapillarity. Philos. Mag., 25, 475-481.

Cherepanov, D. A., Feniouk, B. A., Junge, W. \& Mulkidjanian, A. Y. (2003) Low dielectric permittivity of water at the membrane interface: effect of the energy coupling mechanism in biological membranes. Biophys. J., 85, 1307-1316.

Fromm, J. \& BAUER, T. (1994) Action potentials in maize sieve tubes change phloem translocation. J. Exp. Bot., 45, 463-469.

Gouy, G. (1910) Sur la compression de la charge electrique a la surface d'un electrolyte. J. Phys., 9, 457-468.

Hodgkin, A. L. \& HuXley, A. F. (1952a) The components of membrane conductance in the giant axon of loligo. J. Physiol., 116, 473-496.

Hodgkin, A. L. \& HuXley, A. F. (1952b) Currents carried by sodium and potassium ions through the membrane of the giant axon of loligo. J. Physiol., 116, 449-472.

Hodgkin, A. L. \& Huxley, A. F. (1952c) The dual effect of membrane potential on sodium conductance in the giant axon of loligo. J. Physiol., 116, 497-506.

Hodgkin, A. L. \& Huxley, A. F. (1952d) A quantitative description of membrane current and its application to conduction and excitation in nerve. J. Physiol., 117, 500-544.

Hodgkin, A. L., Huxley, A. F. \& Katz, B. (1952) Measurement of current-voltage relations in the membrane of loligo. J. Physiol., 116, 424-448.

Lindsay, K. A., Rosenburg, J. R. \& Tucker, G. (2004a) From Maxwell's equations to the cable equation and beyond. Prog. Biophys. Mol. Biol., 85, 71-116.

Lindsay, K. A., Rosenburg, J. R. \& Tucker G. (2004b) A note on the discrepancy between the predicted and observed speed of the propagated action potential in the squid giant axon. J. Theor. Biol., 230, 39-48.

LUO, C. H. \& RuDY, Y. (1994) A dynamic model of the cardiac ventricular action potential. I. Simulations of ionic currents and changes. Circ. Res., 74, 1071-1096.

Meunier, C. \& Segev, I. (2002) Playing the devil's advocate: is the Hodgkin-Huxley model useful? Trends Neurosci., 25, 558-563.

Mwesgwa, J., Collins, D. J. \& Volkov, A. G. (1999) Electrochemical signaling in green plans:effect of 2,4-dinitrophenol on variation and action potentials in soybean. Bioelectrochemistry, 51, 201-205.

NEUSS-RADU, M. \& J ̈̈GER, W. (2005) Homogenization of thin porous layers and application to ion transport through channels. Oberwofach Reports.

Newman, J. S. \& Thomas-Alyea, K. E. (2004) Electrochemical Systems, 3rd edn. Wiley-Interscience.

RiChARDSON, G. \& KING, J.R. (2007) Time-dependent modelling and asymptotic analysis of electrochemical cells. J. Eng. Math., 59, 239-275.

\section{Appendix A. The first-order problem in the intermediate region}

Here, we consider the matching conditions that, together with (71-73), lead to a closed problem for the first-order intermediate variables $N_{1}^{(i+)}, K_{1}^{(i+)}$ and $\phi_{1}^{(i+)}$. We can use the solution to the leading order outer potential to evaluate $\partial \phi_{0}^{\text {(out+) }} /\left.\partial \xi\right|_{x=0^{+}}$. In turn, this may be used to determine the outer ion fluxes normal to the membrane

$$
\begin{gathered}
\left.\mathcal{F}_{n, 0}^{(\text {out }+)} \cdot e_{x}\right|_{x=0^{+}}=-\left.D_{n} \bar{N} \frac{\partial \phi_{0}^{(\text {out }+)}}{\partial \xi}\right|_{x=0^{+}},\left.\quad \mathcal{F}_{k, 0}^{(\text {out }+)} \cdot \boldsymbol{e}_{\boldsymbol{x}}\right|_{x=0^{+}}=-\left.D_{k} \bar{K} \frac{\partial \phi_{0}^{(\text {out }+)}}{\partial \xi}\right|_{x=0^{+}}, \\
\left.\mathcal{F}_{c, 0}^{\text {(out }+)} \cdot \boldsymbol{e}_{\boldsymbol{x}}\right|_{x=0^{+}}=\left.D_{c}(\bar{N}+\bar{K}) \frac{\partial \phi_{0}^{(\text {out }+)}}{\partial \xi}\right|_{x=0^{+}} .
\end{gathered}
$$


Matching the leading order outer ion fluxes to the intermediate thus gives

$$
\begin{aligned}
& \lim _{w \rightarrow+\infty} \mathcal{F}_{n x, 0}^{(i+)}=-\left.D_{n} \bar{N} \frac{\partial \phi_{0}^{(\text {out }+)}}{\partial \xi}\right|_{x=0^{+}}, \lim _{w \rightarrow+\infty} \mathcal{F}_{k x, 0}^{(i+)}=-\left.D_{k} \bar{K} \frac{\partial \phi_{0}^{(\text {out }+)}}{\partial \xi}\right|_{x=0^{+}}, \\
& \lim _{w \rightarrow+\infty} \mathcal{F}_{c x, 0}^{(i+)}=\left.D_{c}(\bar{N}+\bar{K}) \frac{\partial \phi_{0}^{(\text {out }+)}}{\partial \xi}\right|_{x=0^{+}}, \lim _{w \rightarrow+\infty} \frac{\partial \phi_{1}^{(i+)}}{\partial w}=\left.\frac{\partial \phi_{0}^{(\text {out }+)}}{\partial x}\right|_{x=0^{+}} .
\end{aligned}
$$

Substituting for $\mathcal{F}_{n x, 0}^{(i+)}, \mathcal{F}_{k x, 0}^{(i+)}$ and $\mathcal{F}_{c x, 0}^{(i+)}$, from (69-70) in the above and simplifying the resulting expressions leads to the following three conditions on $N_{1}^{(i+)}, K_{1}^{(i+)}$ and $\phi_{1}^{(i+)}$ :

$$
\lim _{w \rightarrow+\infty} \frac{\partial N_{1}^{(i+)}}{\partial w}=0, \quad \lim _{w \rightarrow+\infty} \frac{\partial K_{1}^{(i+)}}{\partial w}=0, \quad \lim _{w \rightarrow+\infty} \frac{\partial \phi_{1}^{(i+)}}{\partial w}=\left.\frac{\partial \phi_{0}^{(\text {out }+)}}{\partial x}\right|_{x=0^{+}} .
$$

Matching the leading order ion fluxes in the intermediate to those in the Debye region gives, after some rearrangement,

$$
\begin{aligned}
& \left.\frac{\partial \phi_{1}^{(i+)}}{\partial w}\right|_{w=0}=-\lim _{\xi \rightarrow+\infty} \frac{1}{2(\bar{K}+\bar{N})}\left(\mathcal{F}_{n x, 0}^{(D)}+\mathcal{F}_{k x, 0}^{(D)}+\mathcal{F}_{c x, 0}^{(D)}\right), \\
& \left.\frac{\partial K_{1}^{(i+)}}{\partial w}\right|_{w=0}=-\lim _{\xi \rightarrow+\infty} \frac{1}{2(\bar{K}+\bar{N})}\left(\frac{(2 \bar{N}+\bar{K}) \mathcal{F}_{k x, 0}^{(D)}}{D_{k}}-\frac{\bar{K} \mathcal{F}_{n x, 0}^{(D)}}{D_{n}}+\frac{\bar{K} \mathcal{F}_{c x, 0}^{(D)}}{D_{c}}\right), \\
& \left.\frac{\partial N_{1}^{(i+)}}{\partial w}\right|_{w=0}=-\lim _{\xi \rightarrow+\infty} \frac{1}{2(\bar{K}+\bar{N})}\left(\frac{(2 \bar{K}+\bar{N}) \mathcal{F}_{n x, 0}^{(D)}}{D_{n}}-\frac{\bar{N} \mathcal{F}_{k x, 0}^{(D)}}{D_{k}}+\frac{\bar{N} \mathcal{F}_{c x, 0}^{(D)}}{D_{c}}\right) .
\end{aligned}
$$

Since, as already mentioned, the leading order current density normal to the membrane is independent of $w$ in the intermediate, we have that $\lim _{\xi \rightarrow+\infty} j_{x, 0}^{(D)}=\left.j_{x, 0}^{(\text {out }+)}\right|_{x=0}$. It follows that

$$
\lim _{\xi \rightarrow+\infty}\left(\mathcal{F}_{k x, 0}^{(D)}+\mathcal{F}_{n x, 0}^{(D)}-\mathcal{F}_{c x, 0}^{(D)}\right)=-\left.\left(\bar{K}\left(D_{k}+D_{c}\right)+\bar{N}\left(D_{n}+D_{c}\right)\right) \frac{\partial \phi_{0}^{(\text {out }+)}}{\partial x}\right|_{x=0^{+}}
$$

This, together with (55), gives three relations to determine the three quantities $\lim _{\xi \rightarrow+\infty} \mathcal{F}_{n x, 0}^{(D)}$, $\lim _{\xi \rightarrow+\infty} \mathcal{F}_{k x, 0}^{(D)}$ and $\lim _{\xi \rightarrow+\infty} \mathcal{F}_{n x, 0}^{(D)}$. Once these have been determined, we can substitute the results back into (A.2) to give three conditions on the derivatives of $N_{1}^{(i+)}, K_{1}^{(i+)}$ and $\phi_{1}^{(i+)}$ on $w=0$. These, together with the three corresponding conditions (A.1) at $w=\infty$, form the boundary data for the three second-order equations (71-73) for the intermediate variables $N_{1}^{(i+)}, K_{1}^{(i+)}$ and $\phi_{1}^{(i+)}$. Given the number of boundary conditions and the order of this problem, we might reasonably expect this to be well posed. We thus see that the intermediate provides a mechanism for adjusting the fluxes between the Debye layer and the outer region. 